\title{
A VERY BRIEF INTRODUCTION TO QUANTUM COMPUTING AND QUANTUM INFORMATION THEORY FOR MATHEMATICIANS
}

\author{
J. M. LANDSBERG
}

\begin{abstract}
This is a very brief introduction to quantum computing and quantum information theory, primarily aimed at geometers. Beyond basic definitions and examples, I emphasize aspects of interest to geometers, especially connections with asymptotic representation theory. Proofs of most statements can be found in standard references such as [KSV02, NC00] as well as [Lan17].
\end{abstract}

\section{Overview}

The postulates of quantum mechanics are presented as a generalized probability theory in $\S 2$. In $\S 3$ I describe basic entanglement phenomena of "super dense coding", "teleportation", and Bell's confirmation of the "paradox" proposed by Einstein-Podolsky-Rosen. In $§ 4$ I outline aspects of the basic quantum algorithms, emphasizing the geometry involved. $\S 5$ is a detour into classical information theory, which is the basis of its quantum cousin briefly discussed in $\S 7$. Before that, in $\S 6$, I reformulate quantum theory in terms of density operators, which facilitates the description of quantum information theory. Critical to quantum information theory is von Neumann entropy and in $\S 8$ I elaborate on some of its properties. A generalization of "teleportation" (LOCC) is discussed in $§ 9$. I briefly discuss SLOCC in $\$ 10$ because of its geometric appeal. Regarding practical computation, the exponential growth in size of $\left(\mathbb{C}^{2}\right)^{\otimes n}$ with $n$ that appears in quantum information theory leads to the notion of "feasible" states discussed in $\S 11$, which has interesting algebraic geometry associated to it. I conclude with a discussion of representation-theoretic aspects of quantum information theory, including a discussion of the quantum marginal problem in $\S 12$. I do not discuss topological quantum computing, which utilizes the representation theory of the braid group.

\section{Quantum COMPUtation as Generalized PRobabilistic COMPUTATION}

2.1. Classical and Probabilistic computing via linear algebra. This section is inspired by [AB09, Exercise 10.4].

Classical communication deals with bits, elements of $\{0,1\}$, which will be convenient to think of as elements of $\mathbb{F}_{2}$, the field with two elements. Let $f_{n}: \mathbb{F}_{2}^{n} \rightarrow \mathbb{F}_{2}$ be a sequence of functions. Give $\mathbb{R}^{2}$ basis $\{|0\rangle,|1\rangle\}$ (such notation is standard in quantum mechanics) and give $\left(\mathbb{R}^{2}\right)^{\otimes m}=\mathbb{R}^{2^{m}}$ basis $\left\{|I\rangle \mid I \in\{0,1\}^{m}\right\}$. In this way, we may identify $\mathbb{F}_{2}^{m}$ with the set of basis vectors of $\mathbb{R}^{2^{m}}$. A computation of $f_{n}$ (via an arithmetic or Boolean circuit) may be phrased as a sequence of linear maps on a vector space containing $\mathbb{R}^{2^{n}}$, where each linear map comes from a pre-fixed set agreed

Date: January 19, 2018.

Landsberg supported by NSF grant DMS-1405348. 
upon in advance. In anticipation of what will come in quantum computation, the pre-fixed set of maps will be taken from maps having the following properties:

(1) Each linear map must take probability distributions to probability distributions. This implies the matrices are stochastic: the entries are non-negative and each column sums to 1 .

(2) Each linear map only alters a small number of entries. For simplicity assume it alters at most three entries, i.e., it acts on at most $\mathbb{R}^{2^{3}}$ and is the identity on all other factors in the tensor product.

To facilitate comparison with quantum comptuation, we will restrict to reversible classical computation. The complexity class of a sequence of functions in classical reversible computation is the same as in arbitrary classical computation.

For example, if we want to effect $(x, y) \mapsto x * y$, consider the map

$$
|x, y, z\rangle \mapsto|x, y, z \oplus(x * y)\rangle=|x, y, z \oplus(x \wedge y)\rangle
$$

(where the second expression is for those preferring Boolean notation) and act as the identity on all other basis vectors (sometimes called registers). Here $z$ will represent "workspace bits": $x, y$ will come from the input and $z$ will always be set to 0 in the input. In the basis $|000\rangle,|001\rangle,|010\rangle,|100\rangle,|011\rangle,|101\rangle,|110\rangle,|111\rangle$, of $\mathbb{R}^{8}$, the matrix is

$$
\left(\begin{array}{llllllll}
1 & 0 & 0 & 0 & 0 & 0 & 0 & 0 \\
0 & 1 & 0 & 0 & 0 & 0 & 0 & 0 \\
0 & 0 & 1 & 0 & 0 & 0 & 0 & 0 \\
0 & 0 & 0 & 1 & 0 & 0 & 0 & 0 \\
0 & 0 & 0 & 0 & 1 & 0 & 0 & 0 \\
0 & 0 & 0 & 0 & 0 & 1 & 0 & 0 \\
0 & 0 & 0 & 0 & 0 & 0 & 0 & 1 \\
0 & 0 & 0 & 0 & 0 & 0 & 1 & 0
\end{array}\right)
$$

This gate is sometimes called the Toffoli gate and the matrix the Toffoli matrix.

The swap (negation) gate $\neg$ may be effected by the matrix

$$
\sigma_{x}=\left(\begin{array}{ll}
0 & 1 \\
1 & 0
\end{array}\right)
$$

The swap and Toffoli matrix can perform any computation that is accomplished via a sequence of matrices drawn from some finite set of Boolean operations, each acting on a fixed number of basis vectors with at worst a polynomial in $n$ size increase in the number of matrices needed. For those familiar with Boolean circuits, any sequence of Boolean circuits (one for each $n$ ) may be replaced by a sequence with just Toffoli and negation gates with at worst a polynomial (in $n$ ) blow up in size.

A probability distribution on $\{0,1\}^{m}$ may be encoded as a vector in $\mathbb{R}^{2^{m}}$ : If the probability distribution assigns probability $p_{I}$ to $I \in\{0,1\}^{m}$, assign to the distribution the vector $v=$ $\sum_{I} p_{I}|I\rangle \in \mathbb{R}^{2^{m}}$.

The above matrices realize classical computation. To add randomness to enable probabilistic computation, introduce the matrix

$$
\left(\begin{array}{ll}
\frac{1}{2} & \frac{1}{2} \\
\frac{1}{2} & \frac{1}{2}
\end{array}\right)
$$


which acts on a single $\mathbb{R}^{2}$ corresponding to a fair coin flip. Note that the coin flip matrix is not invertible, which will be one motivation for quantum computation in $\S 2.2$. Work in $\mathbb{R}^{2^{n+s+r}}$ where $r$ is the number of times one needs to access a random choice and $s$ is the number of matrices (arithmetic operations) in addition to the coin tosses needed to compute $f$.

A probabilistic computation, viewed this way, starts with $\left|x 0^{r+s}\right\rangle$, where $x \in \mathbb{F}_{2}^{n}$ is the input. One then applies a sequence of admissible stochastic linear maps to it, and ends with a vector that encodes a probability distribution on $\{0,1\}^{n+s+r}$. One then restricts this to $\{0,1\}^{p(n)}$, that is, one takes the vector and throws away all but the first $p(n)$ entries. This vector encodes a probability sub-distribution, i.e., all coefficients are non-negative and they sum to a number between zero and one. One then renormalizes (dividing each entry by the sum of the entries) to obtain a vector encoding a probability distribution on $\{0,1\}^{p(n)}$ and then outputs the answer according to this distribution. Note that even if our calculation is feasible (i.e., polynomial in size), to write out the original output vector that one truncates would be exponential in cost. A stronger variant of this phenomenon will occur with quantum computing, where the result will be obtained with a polynomial size calculation, but one does not have access to the vector created, even using an exponential amount of computation.

To further prepare for the analogy with quantum computation, define a probabilistic bit (a pbit) to be an element of

$$
\left\{p_{0}|0\rangle+p_{1}|1\rangle \mid p_{j} \in[0,1] \text { and } p_{0}+p_{1}=1\right\} \subset \mathbb{R}^{2} .
$$

Note that the set of pbits (possible states) is a convex set, and the basis vectors are the extremal points of this convex set.

2.2. A wish list. Here is a wish list for how one might want to improve upon the above set-up:

(1) Allow more general kinds of linear maps to get more computing power, while keeping the maps easy to compute.

(2) Have reversible computation: we saw that classical computatation can be made reversible, but the coin flip was not. This property is motivated by physics, where many physical theories require time reversibility.

(3) Again motivated by physics, one would like to have a continous evolution of the probability vector, more precisely, one would like the probability vector to depend on a continuous parameter $t$ such that if $\left|\psi_{t_{1}}\right\rangle=X\left|\psi_{t_{0}}\right\rangle$, then there exist admissible matrices $Y, Z$ such that $\left|\psi_{t_{0}+\frac{1}{2} t_{1}}\right\rangle=Y\left|\psi_{t_{0}}\right\rangle$ and $\left|\psi_{t_{1}}\right\rangle=Z\left|\psi_{t_{0}+\frac{1}{2} t_{1}}\right\rangle$ and $X=Z Y$. In particular, one wants operators to have square roots.

One way to make the coin flip reversible is, instead of making the probability distribution be determined by the sum of the coefficients, one could take the sum of the squares. If one does this, there is no harm in allowing the entries of the output vectors to become negative, and one could use

$$
H:=\frac{1}{\sqrt{2}}\left(\begin{array}{cc}
1 & 1 \\
1 & -1
\end{array}\right)
$$

for the coin flip. The matrix $H$ is called the Hadamard matrix or Hadamard gate in the quantum computing literature. If we make this change, we obtain our second wish, and moreover have many operations be "continous", because the set of matrices preserving the norm-squared of a real-valued vector is the orthogonal group $O(n)=\left\{A \in M a t_{n \times n} \mid A A^{T}=\operatorname{Id}\right\}$. So for example, any rotation has a square root. 
However our third property will not be completely satisfied, as the matrix

$$
\left(\begin{array}{cc}
1 & 0 \\
0 & -1
\end{array}\right)
$$

which represents a reflection, does not have a square root in $O(2)$.

To have the third wish satisfied, allow vectors with complex entries. From now on let $i=\sqrt{-1}$. For a complex number $z=x+i y$ let $\bar{z}=x-i y$ denote its complex conjugate and $|z|^{2}=z \bar{z}$ the square of its norm.

So we go from pbits, $\{p|0\rangle+q|1\rangle \mid p, q \geq 0$ and $p+q=1\}$ to qubits, the set of which is

$$
\left\{\alpha|0\rangle+\beta|1\rangle \mid \alpha, \beta \in \mathbb{C} \text { and }|\alpha|^{2}+|\beta|^{2}=1\right\} .
$$

The set of qubits, considered in terms of real parameters, looks at first like the 3 -sphere $S^{3}$ in $\mathbb{R}^{4} \simeq \mathbb{C}^{2}$. However, the probability distributions induced by $|\psi\rangle$ and $e^{i \theta}|\psi\rangle$ are the same so it is really $S^{3} / S^{1}$ (the Hopf fibration), i.e., the two-sphere $S^{2}$. Geometrically, it would be more natural (especially since we have already seen the need to re-normalize in probabilistic computation) to work with projective space $\mathbb{C P}^{1} \simeq S^{2}$ as our space of qubits, instead of a subset of $\mathbb{C}^{2}$. So the set of qubits is better seen as (4) modulo the equivalence $|\psi\rangle \sim e^{i \theta}|\psi\rangle$.

For $v=\left(v_{1}, \cdots, v_{n}\right) \in \mathbb{C}^{n}$, write $|v|^{2}=\left|v_{1}\right|^{2}+\cdots+\left|v_{n}\right|^{2}$. The set of stochastic matrices is now replaced by the unitary group

$$
\left.\mathbf{U}(n):=\left\{A \in \operatorname{Mat}_{n \times n}(\mathbb{C})|| A v|=| v|\forall| v\right\rangle \in \mathbb{C}^{n}\right\} .
$$

The unitary group satisfies the third wish on the list: For all $A \in \mathbf{U}(n)$, there exists a matrix $B \in \mathbf{U}(n)$ satisfying $B^{2}=A$.

Consider wish 1: it is an open question! However at least our generalized probabilistic computation includes our old probabilistic computation because $H$ and the matrices (2), (3) are unitary.

An indication that generalized probability may be related to quantum mechanics is that the interference patterns observed in the famous two slit experiments is manifested in generalized probability: one obtains a "random bit" by applying $H$ to $|0\rangle: H|0\rangle=\frac{1}{\sqrt{2}}(|0\rangle+|1\rangle)$. However, if one applies a second quantum coin flip, one looses the randomness as $H^{2}|0\rangle=|1\rangle$, which, as pointed out in [Aar13], could be interpreted as a manifestation of interference.

2.3. Postulates of quantum mechanics and relevant linear algebra. Here are the standard postulates of quantum mechanics and relevant definitions from linear algebra.

P1. Associated to any isolated physical system is a Hilbert space $\mathcal{H}$, called the state space. The system is completely described at a given moment by a unit vector $|\psi\rangle \in \mathcal{H}$, called its state vector, which is well defined up to a phase $e^{i \theta}$ with $\theta \in \mathbb{R}$. Alternatively one may work in projective space $\mathbb{P H}$.

Explanations. A Hilbert space $\mathcal{H}$ is a (complete) complex vector space endowed with a nondegenerate Hermitian inner-product, $h: \mathcal{H} \times \mathcal{H} \rightarrow \mathbb{C}$, where by definition $h$ is linear in the first factor and conjugate linear in the second, $h(|v\rangle,|w\rangle)=\overline{h(|w\rangle,|v\rangle)}$ for all $v, w$, and $h(|v\rangle,|v\rangle)>0$ for all $|v\rangle \neq 0$. 


\section{Remarks:}

When studying quantum mechanics in general, one needs to allow infinite dimensional Hilbert spaces, but in the case of quantum computing, one restricts to finite dimensional Hilbert spaces, usually $\left(\mathbb{C}^{2}\right)^{\otimes N}$.

The Hermitian inner-product $h$ allows an identification of $\mathcal{H}$ with $\mathcal{H}^{*}$ by $|w\rangle \mapsto\langle w|:=h(\cdot,|w\rangle)$. This identification will be used repeatedly. Write $h(|v\rangle,|w\rangle)=\langle w \mid v\rangle$ and $|v|=\sqrt{\langle v \mid v\rangle}$ for the length of $|v\rangle$.

If $\mathcal{H}=\mathbb{C}^{n}$ with its standard basis, where $|v\rangle=\left(v_{1}, \cdots, v_{n}\right)$, the standard Hermitian inner-product on $\mathbb{C}^{n}$ is $\langle w \mid v\rangle=\sum_{j=1}^{n} \bar{w}_{j} v_{j}$. I will always assume $\mathbb{C}^{n}$ is equipped with its standard Hermitian inner-product.

P2. The state of an isolated system evolves with time according to the Schrödinger equation

$$
i \hbar \frac{d|\psi\rangle}{d t}=X|\psi\rangle
$$

where $\hbar$ is a constant (Planck's constant) and $X$ is a fixed Hermitian operator, called the Hamiltonian of the system. Here, recall that the adjoint of an operator $X \in \operatorname{End}(\mathcal{H})$, is the operator $X^{\dagger} \in \operatorname{End}(\mathcal{H})$ such that $\left\langle X^{\dagger} v \mid w\right\rangle=\langle v \mid X w\rangle$ for all $v, w \in \mathcal{H}$ and $X$ is Hermitian if $X=X^{\dagger}$. For a general Hilbert space, the Unitary group is $\mathbf{U}(\mathcal{H}):=\{U \in \operatorname{End}(\mathcal{H})|| U v|=| v|\forall| v\rangle \in \mathcal{H}\}$.

How is generalized probability related to Schrödinger's equation? Let $U(t) \subset \mathbf{U}(\mathcal{H})$ be a smooth curve with $U(0)=\mathrm{Id}$. Write $U^{\prime}(0)=\left.\frac{d}{d t}\right|_{t=0} U(t)$. Consider

$$
\begin{aligned}
0 & =\left.\frac{d}{d t}\right|_{t=0}\langle v \mid w\rangle \\
& =\left.\frac{d}{d t}\right|_{t=0}\langle U(t) v \mid U(t) w\rangle \\
& =\left\langle U^{\prime}(0) v \mid w\right\rangle+\left\langle v \mid U^{\prime}(0) w\right\rangle .
\end{aligned}
$$

Thus $i U^{\prime}(0)$ is Hermitian. We are almost at Schrödinger's equation. Let $\mathfrak{u}(\mathcal{H})=T_{\mathrm{Id}} \mathbf{U}(\mathcal{H})$ denote the Lie algebra of $\mathbf{U}(\mathcal{H})$ so $i \mathfrak{u}(\mathcal{H})$ is the space of Hermitian endomorphisms. For $X \in \operatorname{End}(\mathcal{H})$, write $X^{k} \in \operatorname{End}(\mathcal{H})$ for $X \cdots X$ applied $k$ times. Write $e^{X}:=\sum_{k=0}^{\infty} \frac{1}{k !} X^{k}$. If $X$ is Hermitian, then $e^{i X} \in \mathbf{U}(\mathcal{H})$. Postulate 2 implies the system will evolve unitarily, by (assuming one starts at $t=0),\left|\psi_{t}\right\rangle=U(t)\left|\psi_{0}\right\rangle$, where

$$
U(t)=e^{\frac{-i t X}{h}} .
$$

Measurements. Our first two postulates dealt with isolated systems. In reality, no system is isolated and the whole universe is modeled by one enormous Hilbert space. In practice, parts of the system are sufficiently isolated that they can be treated as isolated systems. However, they are occasionally acted upon by the outside world, and one needs a way to describe this outside interference. For our purposes, the isolated systems will be the Hilbert space attached to the input in a quantum algorithm and the outside interference will be the measurement at the end. That is, after a sequence of unitary operations one obtains a vector $|\psi\rangle=\sum z_{j}|j\rangle$ (here implicitly assuming the Hilbert space is of countable dimension), and as in generalized probability:

P3 The probability of obtaining outcome $j$ under a measurement equals $\left|z_{j}\right|^{2}$. 
In $\S 6$, motivated again by probability, P1,P3 will be generalized to new postulates that give rise to the same theory, but are more convenient to work with in information theory.

A typical situation in quantum mechanics and quantum computing is that there are two or more isolated systems, say $\mathcal{H}_{A}, \mathcal{H}_{B}$ that are brought together (i.e., allowed to interact with each other) to form a larger isolated system $\mathcal{H}_{A B}$. The larger system is called the composite system. In classical probability, the composite space is $\{0,1\}^{N_{A}} \times\{0,1\}^{N_{B}}$. In our generalized probability, the composite space is $\left(\mathbb{C}^{2}\right)^{\otimes N_{A}} \otimes\left(\mathbb{C}^{2}\right)^{\otimes N_{B}}=\left(\mathbb{C}^{2}\right)^{\otimes\left(N_{A}+N_{B}\right)}$ :

P4: The state of a composite system $\mathcal{H}_{A B}$ is the tensor product of the state spaces of the component physical systems $\mathcal{H}_{A}, \mathcal{H}_{B}: \mathcal{H}_{A B}=\mathcal{H}_{A} \otimes \mathcal{H}_{B}$.

When dealing with composite systems, we will need to allow partial measurements whose outcomes are of the form $|I\rangle \otimes|\phi\rangle$ with $|\phi\rangle$ arbitrary.

This tensor product structure gives rise to the notion of entanglement, which accounts for phenomenon outside of our classical intuition, as discussed in the next section.

Definition 2.1. A state $|\psi\rangle \in \mathcal{H}_{1} \otimes \cdots \otimes \mathcal{H}_{n}$ is called separable if it corresponds to a rank one tensor, i.e., $|\psi\rangle=\left|v_{1}\right\rangle \otimes \cdots \otimes\left|v_{n}\right\rangle$ with each $\left|v_{j}\right\rangle \in \mathcal{H}_{j}$. Otherwise it is entangled.

\section{Entanglement Phenomena}

3.1. Super-dense coding. ${ }^{1}$ Physicists describe their experiments in terms of two characters, Alice and Bob. I generally follow this convention. Let $\mathcal{H}=\mathbb{C}^{2} \otimes \mathbb{C}^{2}=\mathcal{H}_{A} \otimes \mathcal{H}_{B}$, and let $\mid$ epr $\rangle=$ $\frac{|00\rangle+|11\rangle}{\sqrt{2}}$ (called the EPR state in the physics literature after Einstein-Podolsky-Rosen). Assume this state has been created, both Alice and Bob are aware of it, Alice is in possession of the first qubit, and Bob the second. In particular Alice can act on the first qubit by unitary matrices and Bob can act on the second. This all happens before the experiment begins.

Now say Alice wants to transmit a two classical bit message to Bob, i.e., one of the four states $|00\rangle,|01\rangle,|10\rangle,|11\rangle$ by transmitting qubits. We will see that she can do so transmitting just one qubit. If she manipulates her qubit by acting on the first $\mathbb{C}^{2}$ by a unitary transformation, $|e p r\rangle$ will be manipulated. She uses the following matrices depending on the message she wants to transmit:

\begin{tabular}{|r|c|c|}
\hline to transmit & act by & to obtain \\
\hline$|00\rangle$ & Id & $\frac{|00\rangle+|11\rangle}{\sqrt{2}}$ \\
$|01\rangle$ & $\left(\begin{array}{cc}1 & 0 \\
0 & -1\end{array}\right)=: \sigma_{z}$ & $\frac{|00\rangle-|11\rangle}{\sqrt{2}}$ \\
$|10\rangle$ & $\left(\begin{array}{cc}0 & 1 \\
1 & 0\end{array}\right)=: \sigma_{x}$ & $\frac{|10\rangle+|01\rangle}{\sqrt{2}}$ \\
$|11\rangle$ & $\left(\begin{array}{cc}0 & -1 \\
1 & 0\end{array}\right)=:-i \sigma_{y}$ & $\frac{|01\rangle-|10\rangle}{\sqrt{2}}$
\end{tabular}

where the names $\sigma_{x}, \sigma_{y}, \sigma_{z}$ are traditional in the physics literature (the Pauli matrices). If Alice sends Bob her qubit, so he is now in possession of the modified $|e p r\rangle$ (although he does not see it), he can determine which of the four messages she sent him by measuring $|e p r\rangle$. More precisely, first Bob acts on $\mathbb{C}^{2} \otimes \mathbb{C}^{2}$ by a unitary transformation that takes the orthonormal basis in the "to obtain" column to the standard orthonormal basis (this is a composition of the inverses of two

\footnotetext{
${ }^{1}$ Physicists use the word "super" in the same way American teenagers use the word "like".
} 
Hadamard matrices), to obtain a state vector whose probability is concentrated at one of the four classical states, then he measures, and obtains the correct classical state with probability one.

In summary, with preparation of an EPR state in advance, plus transmission of a single qubit, one can transmit two classical bits of information.

3.2. Quantum teleportation. A similar phenomenon is the misleadingly named quantum teleportation, where again Alice and Bob share half of an EPR state, Alice is in possession of a qubit $|\psi\rangle=\alpha|0\rangle+\beta|1\rangle$, and wants to "send" $|\psi\rangle$ to Bob. However Alice is only allowed to transmit classical information to Bob (in particular, that information is transmitted at a speed slower than the speed of light, which is why the use of the word "teleportation" is misleading). We will see that she can transmit a qubit to Bob by transmitting two classical bits. Write the state of the system as

$$
\frac{1}{\sqrt{2}}[\alpha|0\rangle \otimes(|00\rangle+|11\rangle)+\beta|1\rangle \otimes(|00\rangle+|11\rangle)]
$$

where Alice can operate on the first two qubits. If Alice acts on the first two qubits by $H \otimes \sigma_{x}=$ $\frac{1}{\sqrt{2}}\left(\begin{array}{cc}1 & 1 \\ 1 & -1\end{array}\right) \otimes\left(\begin{array}{ll}0 & 1 \\ 1 & 0\end{array}\right)$, she obtains

$$
\frac{1}{2}[|00\rangle \otimes(\alpha|0\rangle+\beta|1\rangle)+|01\rangle \otimes(\alpha|1\rangle+\beta|0\rangle)+|10\rangle \otimes(\alpha|0\rangle-\beta|1\rangle)+|11\rangle \otimes(\alpha|1\rangle-\beta|0\rangle)] .
$$

Notice that Bob's coefficient of Alice's $|00\rangle$ is the state $|\psi\rangle$ that is to be transmitted. Alice performs a measurement. If she has the good luck to obtain $|00\rangle$, then she knows Bob has $|\psi\rangle$ and she can tell him classically that he is in possession of $|\psi\rangle$. But say she obtains the state $|01\rangle$ : the situation is still good, she knows Bob is in possession of a state such that, if he acts on it with $\sigma_{x}=\left(\begin{array}{ll}0 & 1 \\ 1 & 0\end{array}\right)$, he will obtain the state $|\psi\rangle$, so she just needs to tell him classically to apply $\sigma_{x}$. Since they had communicated the algorithm in the past, all Alice really needs to tell Bob in the first case is the classical message 00 and in the second case the message 01 . The cases of 10 and 11 are similar.

In summary, a shared EPR pair plus sending two classical bits of information allows transmission of one qubit.

3.3. Bell's game. The 1935 Einstein-Podolsky-Rosen paper [EPR35] challenged quantum mechanics with the following thought experiment that they believed implied instantaneous communication across distances, in violation of principles of relativity: Alice and Bob prepare $|e p r\rangle=\frac{1}{\sqrt{2}}(|00\rangle+|11\rangle)$, then travel far apart. Alice measures her bit. If she gets 0 , then she can predict with certainty that Bob will get 0 in his measurement, even if his measurement is taken a second later and they are a light year apart.

Ironically, this thought experiment has been made into an actual experiment. One modern interpretation (see, e.g., [AB09]) is that there is no paradox because the system does not transmit information faster than the speed of light, but rather they are acting on information that has already been shared. What follows is a version from [CHSH69], adapted from the presentation in $[\mathrm{AB} 09]$.

Charlie chooses $x, y \in\{0,1\}$ at random and sends $x$ to Alice and $y$ to Bob. Based on this information, Alice and Bob, without communicating with each other, get to choose bits $a, b$ 
and send them to Charlie. The game is such that Alice and Bob play on a team. They win if $a \oplus b=x \wedge y$, i.e., either $(x, y) \neq(1,1)$ and $a=b$ or $(x, y)=(1,1)$ and $a \neq b$.

3.3.1. Classical version. Note that if Alice and Bob both always choose 0, they win with probability $\frac{3}{4}$.

Theorem 3.1. [Bel64] Regardless of the strategy Alice and Bob use, they never win with probability greater than $\frac{3}{4}$.

3.3.2. Quantum version. Although there is still no communication allowed between Alice and Bob, they will exploit a pre-shared $|e p r\rangle$ to gain an advantage over the classical case. Alice and Bob prepare $|e p r\rangle=\frac{|00\rangle+|11\rangle}{\sqrt{2}}$ in advance, and Alice takes the first qubit and Bob the second. When Alice gets $x$ from Charlie, if $x=1$, she applies a rotation by $\frac{\pi}{8}$ to her qubit, and if $x=0$ she does nothing. When Bob gets $y$ from Charlie, he applies a rotation by $-\frac{\pi}{8}$ to his qubit if $y=1$ and if $y=0$ he does nothing. (The order these rotations are applied does not matter because the corresponding operators on $\left(\mathbb{C}^{2}\right)^{\otimes 2}$ commute.) Both of them measure their respective qubits and send the values obtained to Charlie.

Theorem 3.2. With this strategy, Alice and Bob win with probability at least $\frac{4}{5}$.

The idea behind the strategy is that when $(x, y) \neq(1,1)$, the states of the two qubits will have an angle at most $\frac{\pi}{8}$ between them, but when $(x, y)=(1,1)$, the angle will be $\frac{\pi}{4}$.

\section{Quantum Algorithms}

4.1. Grover's search algorithm. The problem: given $F_{n}: \mathbb{F}_{2}^{n} \rightarrow \mathbb{F}_{2}$, computable by a poly $(n)$ size classical circuit, find $a$ such that $F_{n}(a)=1$ if such $a$ exists.

Grover found a quantum circuit of size poly $(n) 2^{\frac{n}{2}}$ that solves this problem with high probability. Compare this with a brute force search, which requires a circuit of size poly $(n) 2^{n}$. No classical or probabilistic algorithm is known that does better than poly $(n) 2^{n}$. Note that it also gives a size poly $(n) 2^{\frac{n}{2}}$ probabilistic solution to the NP-complete problem SAT (it is stronger, as it not only determines existence of a solution, but finds it).

I present the algorithm for the following simplified version where one is promised there exists exactly one solution. All essential ideas of the general case are here.

Problem: given $F_{n}: \mathbb{F}_{2}^{n} \rightarrow \mathbb{F}_{2}$, computable by a poly $(n)$-size classical circuit, and the information that there is exactly one vector $a$ with $F_{n}(a)=1$, find $a$.

The idea of the algorithm is to start with a vector equidistant from all possible solutions, and then to incrementally rotate it towards $a$. What is strange for our classical intuition is that one is able to rotate towards the solution without knowing what it is, and similarly, we won't "see" the rotation matrix either.

Work in $\left(\mathbb{C}^{2}\right)^{\otimes n+s}$ where $s=s(n)$ is the size of the classical circuit needed to compute $F_{n}$. I suppress reference to the $s$ "workspace bits" in what follows.

The following vector is the average of all the classical (observable) states:

$$
|a v\rangle:=\frac{1}{2^{\frac{n}{2}}} \sum_{I \in\{0,1\}^{n}}|I\rangle .
$$


To prepare $|a v\rangle$, note that $H|0\rangle=\frac{1}{\sqrt{2}}(|0\rangle+|1\rangle)$, so applying $H^{\otimes n}$ to $|0 \cdots 0\rangle$ transforms it to $|a v\rangle$. The cost of this is $n$ gates (matrices).

Since $|a v\rangle$ is equidistant from all possible solution vectors, $\langle a v \mid a\rangle=\frac{1}{2^{\frac{n}{2}}}$. We want to rotate $|a v\rangle$ towards the unknown $a$. Recall that $\cos (\angle(|v\rangle,|w\rangle))=\frac{\langle v \mid w\rangle}{|v||w|}$. Write the angle between $a v$ and $a$ as $\frac{\pi}{2}-\theta$, so $\sin (\theta)=\frac{1}{2^{\frac{n}{2}}}$.

A rotation is a product of two reflections. In order to perform the rotation $R$ that moves $|a v\rangle$ towards $|a\rangle$, first reflect in the hyperplane orthogonal to $|a\rangle$, and then in the hyperplane orthogonal to $|a v\rangle$.

Consider the map

$$
|x y\rangle \mapsto|x(y \oplus F(x))\rangle
$$

defined on basis vectors and extended linearly. To execute this, use the $s$ workspace bits that are suppressed from the notation, to effect $s$ reversible classical gates. Initially set $y=0$ so that the image is $|x 0\rangle$ for $x \neq a$, and $|x 1\rangle$ when $x=a$. Next apply the quantum gate $\operatorname{Id} \otimes\left(\begin{array}{cc}1 & 0 \\ 0 & -1\end{array}\right)$ which sends $|x 0\rangle \mapsto|x 0\rangle$, and $|x 1\rangle \mapsto-|x 1\rangle$. Finally apply the map $|x y\rangle \mapsto|x(y \oplus F(x))\rangle$ again.

Thus $|a 0\rangle \mapsto-|a 0\rangle$ and all other vectors $|b 0\rangle$ are mapped to themselves, as desired.

Next we need to reflect around $|a v\rangle$. It is easy to reflect around a classical state, so first perform the map $H^{-1 \otimes n}$ that sends $|a v\rangle$ to $|0 \cdots 0\rangle$, then reflect in the hyperplane perpendicular to $|0 \cdots 0\rangle$ using the Boolean function $g: \mathbb{F}_{2}^{n} \rightarrow \mathbb{F}_{2}$ that outputs 1 if and only if its input is $(0, \cdots, 0)$, in the role of $F$ for our previous reflection, then apply Hadamard again so the resulting reflection is about $|a v\rangle$.

The composition of these two reflections is the desired rotation $R$. The vector $R|a v\rangle$ is not useful as measuring it only slightly increases the probability of obtaining $|a\rangle$, but if one composes $R$ with itself $O\left(\frac{1}{\theta}\right)$ times, one obtains a vector much closer to $|a\rangle$. (Note that $\theta \sim \sin (\theta)$ so $\frac{1}{\theta} \sim \sqrt{N}$.)

4.2. The quantum discrete Fourier transform. Underlying the famous quantum algorithm of Shor for factoring integers and Simon's algorithm that led up to it, are "quantum" versions of the discrete Fourier transform on finite abelian groups.

The DFT for $\mathbb{Z} / M \mathbb{Z}$, in vector notation, for $j \in \mathbb{Z} / M \mathbb{Z}$, is

$$
|j\rangle \mapsto \frac{1}{\sqrt{M}} \sum_{k=0}^{M-1} \omega^{j k}|k\rangle
$$

where $\omega=e^{\frac{2 \pi i}{M}}$. It is a unitary change of basis such that in the new basis, multiplication in $\mathbb{Z} / M \mathbb{Z}$ is given by a diagonal matrix, and the classical FFT writes the DFT as a product of $O(\log (M))$ sparse matrices (each with $M<M^{2}$ nonzero entries), for a total cost of $O(\log (M) M)<O\left(M^{2}\right)$ arithmetic operations.

Write $M=2^{m}$. The DFT can be written as a product of $O\left(m^{3}\right)=O\left(\log (M)^{3}\right)$ controlled local unitary operators. Hence one can approximately obtain the output vector by a sequence of $\operatorname{poly}(m)$ unitary operators from our gate set with the caveat that we won't be able to "see" it. 
Here is the quantum DFT: It will be convenient to express $j$ in binary and view $\mathbb{C}^{M}=\left(\mathbb{C}^{2}\right)^{\otimes m}$, i.e., write

$$
|j\rangle=\left|j_{1}\right\rangle \otimes \cdots \otimes\left|j_{m}\right\rangle
$$

where $j=j_{1} 2^{m-1}+j_{2} 2^{m-2}+\cdots+j_{m} 2^{0}$ and $j_{i} \in\{0,1\}$. Write the DFT as

$$
\begin{aligned}
& \left|j_{1}\right\rangle \otimes \cdots \otimes\left|j_{m}\right\rangle \\
& \mapsto \frac{1}{\sqrt{M}} \sum_{k=0}^{M-1} \omega^{j k}|k\rangle \\
& =\frac{1}{\sqrt{M}} \sum_{k_{i} \in\{0,1\}} \omega^{j\left(\sum_{l=1}^{m} k_{l} 2^{m-l}\right)}\left|k_{1}\right\rangle \otimes \cdots \otimes\left|k_{m}\right\rangle \\
& =\frac{1}{\sqrt{M}} \sum_{k_{i} \in\{0,1\}} \bigotimes_{l=1}^{m}\left[\omega^{j k_{l} 2^{m-l}}\left|k_{l}\right\rangle\right] \\
& =\frac{1}{\sqrt{M}} \sum_{k_{i} \in\{0,1\}} \bigotimes_{l=1}^{m}\left[\omega^{\left(j_{1} 2^{2 m-1-l}+\cdots+j_{m} 2^{m-l}\right) k_{l}}\left|k_{l}\right\rangle\right] \\
& =\frac{1}{2^{\frac{m}{2}}}\left(|0\rangle+\omega^{j_{m} 2^{-1}}|1\rangle\right) \otimes\left(|0\rangle+\omega^{j_{m-1} 2^{-1}+j_{m} 2^{-2}}|1\rangle\right) \otimes\left(|0\rangle+\omega^{j_{m-2} 2^{-1}+j_{m-1} 2^{-2}+j_{m} 2^{-3}}|1\rangle\right) \\
& \otimes \cdots \otimes\left(|0\rangle+\omega^{\sum_{s=0}^{m-1} j_{m-s} 2^{m-(s+1)}}|1\rangle\right)
\end{aligned}
$$

where for the last line if $2 m-s-l>m$, i.e., $s+l<m$, there is no contribution with $j_{s}$ because $\omega^{2^{m}}=1$, and I multiplied all terms by $1=\omega^{2^{-m}}$ to have negative exponents.

It will be notationally more convenient to write the quantum circuit for this vector with the order of factors reversed, so I describe a quantum circuit that produces

$$
\begin{aligned}
& \frac{1}{\sqrt{2}}\left(|0\rangle+\omega^{\sum_{s=0}^{m-1} j_{m-s} 2^{m-(s+1)}}|1\rangle\right) \otimes \cdots \otimes \frac{1}{\sqrt{2}}\left(|0\rangle+\omega^{j_{m-2} 2^{-1}+j_{m-1} 2^{-2}+j_{m} 2^{-3}}|1\rangle\right) \\
& \otimes \frac{1}{\sqrt{2}}\left(|0\rangle+\omega^{j_{m-1} 2^{-1}+j_{m} 2^{-2}}|1\rangle\right) \otimes \frac{1}{\sqrt{2}}\left(|0\rangle+\omega^{j_{m} 2^{-1}}|1\rangle\right) .
\end{aligned}
$$

Set

$$
R_{k}=\left(\begin{array}{cc}
1 & 0 \\
0 & \omega^{2^{k}}
\end{array}\right),
$$

then (8) is obtained as follows: first apply $H$ to $\left(\mathbb{C}^{2}\right)_{1}$ then a linear map $\Lambda^{1} R_{j}$, defined by $|x\rangle \otimes|y\rangle \mapsto|x\rangle \otimes R_{j}|y\rangle$ if $|x\rangle \neq|0\rangle$ and to $|x\rangle \otimes|y\rangle$ if $|x\rangle=|0\rangle$, to $\left(\mathbb{C}^{2}\right)_{j} \otimes\left(\mathbb{C}^{2}\right)_{1}$ for $j=2, \cdots, m$. Note that at this point only the $\left(\mathbb{C}^{2}\right)_{1}$-term has been altered. From now on leave the $\left(\mathbb{C}^{2}\right)_{1}$-slot alone. Next apply $H$ to $\left(\mathbb{C}^{2}\right)_{2}$ then $\Lambda^{1} R_{j-1}$ to $\left(\mathbb{C}^{2}\right)_{j} \otimes\left(\mathbb{C}^{2}\right)_{2}$ for $j=3, \cdots, m$. Then apply $H$ to $\left(\mathbb{C}^{2}\right)_{3}$ then $\Lambda^{1} R_{j-2}$ to $\left(\mathbb{C}^{2}\right)_{j} \otimes\left(\mathbb{C}^{2}\right)_{3}$ for $j=4, \cdots, m$. Continue, until finally one just applies $H$ to $\left(\mathbb{C}^{2}\right)_{m}$. Finally to obtain the DFT, reverse the orders of the factors (a classical operation).

In practice, one has to fix a quantum gate set, i.e., a finite set of unitary operators that will be allowed in algorithms, in advance, so in general it will be necessary to approximate the transformations $R_{k}$ from elements of our gate set, so one only obtains an approximation of the DFT. 
4.3. The hidden subgroup problem. Given a discrete group $G$ with a specific representation of its elements in binary, a function $f: G \rightarrow \mathbb{F}_{2}^{n}$, and a device that computes $f$ (for unit cost), and the knowledge that there exists a subgroup $G^{\prime} \subset G$ such that $f(x)=f(y)$ if and only if $x y^{-1} \in G^{\prime}$, find $G^{\prime}$.

For finitely generated abelian groups, it is sufficient to solve the problem for $G=\mathbb{Z}^{\oplus k}$ as all finitely generated abelian groups are quotients of some $\mathbb{Z}^{\oplus k}$.

Simons algorithm is for the hidden subgroup problem with $G=\mathbb{Z}_{2}^{\oplus m}$. The $D F T_{2}$ matrix is just

$$
H=\frac{1}{\sqrt{2}}\left(\begin{array}{cc}
1 & -1 \\
-1 & 1
\end{array}\right)
$$

and $G^{\prime}$ is the subgroup generated by $a \in \mathbb{Z}_{2}^{\oplus m}$.

Shor's algorithm for factoring (after classical preparation) amounts to the case $G=\mathbb{Z}$ and $F$ is the function $x \mapsto a^{x} \bmod N$.

\section{Classical information theory}

Quantum information theory is based on classical information theory, so I review the classical theory. The discovery/invention of the bit by Tukey and its development by Shannon [Sha48] was one of the great scientific achievements of the twentieth century, as it changed the way one views information, giving it an abstract formalism that is discussed in this section. The link to quantum information is explained in $\S 7$.

5.1. Data compression: noiseless channels. (Following [BCHW16]) A source emits symbols $x$ from an alphabet $\mathcal{X}$ that we want to store efficiently so we try to encode $x$ in a small number of bits, to say $y \in \mathcal{Y}$ in a way that one can decode it later to recover $x$.

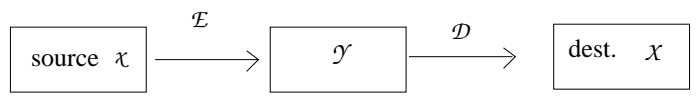

Figure 1. Message from source encoded into bits then decoded

The symbols from $\mathcal{X}$ do not necessarily occur with the same frequency. Let $p=P_{\mathcal{X}}$ denote the associated probability distribution. What is the minimum possible size for $\mathcal{Y}$ ? Since we are dealing in bits, it will be convenient to use the logarithms of cardinalities, so define the capacity as $\operatorname{Cap}\left(P_{\mathcal{X}}\right):=\min \log |\mathcal{Y}|$.

Consider the case $\mathcal{X}=\{a, b, c, d\}$ where $p(a)=0.1, p(b)=0, p(c)=0.4$ and $p(d)=0.5$. One can clearly get away with $|\mathcal{Y}|=3$, e.g., for the encoder, send $a, b$ to $1, c$ to 2 and $d$ to 3 , then for the decoder, send 1 to $a, 2$ to $c$ and 3 to $d$. In general, one can always throw away symbols with probability zero. On the other hand, one cannot map two distinct symbols that do occur to the same symbol, as there would be no way to distinguish them when decoding. Thus $\operatorname{Cap}(p)=\log \operatorname{supp}(p)$, where $\operatorname{supp}(p)=\#\{x \in \mathcal{X} \mid p(x)>0\}$.

Now say we are willing to tolerate a small error. First rephrase what we did probabilistically: Let $p^{e n c}(y \mid x)$ denote the conditional probability distribution of the encoder $\mathcal{E}$ and $p^{\operatorname{dec}}(x \mid y)$ that of the decoder $\mathcal{D}$. Our requirement was for all $x$,

$$
p[x=\mathcal{D} \circ \mathcal{E}(x)]=\sum_{y, x^{\prime}} p^{e n c}(y \mid x) p^{\operatorname{dec}}\left(x^{\prime} \mid y\right) \delta_{x, x^{\prime}}=1 .
$$


Now relax it to

$$
\sum_{x, y, x^{\prime}} p(x) p^{e n c}(y \mid x) p^{d e c}\left(x^{\prime} \mid y\right) \delta_{x, x^{\prime}} \geq 1-\epsilon .
$$

for some error $\epsilon$ that we are willing to tolerate. In addition to throwing out the symbols that do not appear, we may also discard the largest set of symbols whose total probability is smaller than $\epsilon$. Call the corresponding quantity $\operatorname{Cap}^{\epsilon}(p)$.

In the example above, if one takes $\epsilon>0.1$, one can lower storage cost, taking $|\mathcal{Y}|=2$.

Recall that a probability distribution $p$ on $\mathcal{X}$ must satisfy $\sum_{x \in \mathcal{X}} p(x)=1$. Relax this to nonnormalized probability distributions, $q$, where $q(x) \geq 0$ for all $x \in \mathcal{X}$ and $\sum_{x \in \mathcal{X}} q(x) \leq 1$. We obtain: $\operatorname{Cap}^{\epsilon}(p)=\min \log \operatorname{supp}(q)$, where the min is taken over all non-normalized probability distributions $q$ satisfying $q(x) \leq p(x)$ and $\sum_{x \in \mathcal{X}} q(x) \geq 1-\epsilon$.

Now say we get not a single symbol, but a string of $n$ symbols, so we seek an encoder $\mathcal{E}: \mathcal{X}^{n} \rightarrow$ $\mathcal{Y}(n)$, where $\mathcal{Y}(n)$ is a set that varies with $n$, and decoder $\mathcal{D}: \mathcal{Y}(n) \rightarrow \mathcal{X}^{n}$, and we want to minimize $|\mathcal{Y}(n)|$, with a tolerance of error that goes to zero as $n$ goes to infinity. In practice one wants to send information through a communication channel (e.g. telegraph wire). The channel can only send a limited number of bits per second, and we want to maximize the amount of information we can send per second. Define $\operatorname{Rate}(p):=\lim _{\epsilon \rightarrow 0} \lim _{n \rightarrow \infty} \frac{1}{n} \operatorname{Cap}^{\epsilon}\left(p^{n}\right)$.

The string $x_{1} \cdots x_{n}=: \bar{x}^{n}$ is identically and independently distributed (i.i.d), that is each $x_{j}$ is drawn from the same probability distribution and the draw of $x_{j}$ is independent of the draws of the other $x_{i}$. Say $\mathcal{X}=\{1, \cdots, d\}$ with $p(j)=p_{j}$. The probability of any given string occurring depends only on the number of 1's 2's etc.. in the string and not on their order. A string with $c_{j} j$ 's occurs with probability $p_{1}^{c_{1} \cdots} p_{d}^{c_{d}}$. (Note that $c_{1}+\cdots+c_{d}=n$.) The number of strings with this probability is

$$
\left(\begin{array}{c}
n \\
c_{1}, \cdots, c_{d}
\end{array}\right):=\frac{n !}{c_{1} ! \cdots c_{d} !}
$$

and we need to estimate this quantity.

Stirling's formula implies $\log (n !)=n \log (n)-n+O(\log (n))$. In particular, for $0<\beta<1$ such that $\beta n \in \mathbb{Z}$,

$$
\log \left(\begin{array}{c}
n \\
\beta n
\end{array}\right)=n[-\beta \log (\beta)-(1-\beta) \log (1-\beta)]+O(\log (n)) .
$$

Let $H(\beta)=-\beta \log (\beta)-(1-\beta) \log (1-\beta)$ and more generally, for $\bar{p}=\left(p_{1}, \cdots, p_{d}\right)$, let

$$
H(\bar{p})=-\sum_{i=1}^{d} p_{i} \log \left(p_{i}\right),
$$

the Shannon entropy of $\bar{p}$. It plays a central role in information theory.

Define a map $w t: \mathcal{X}^{n} \rightarrow \mathbb{R}^{d}$ by $\bar{x}^{n} \mapsto\left(c_{1}, \cdots, c_{d}\right)$, where $c_{j}$ is the number of $j$ 's appearing in $\bar{x}^{n}$. Then the expectation is $E\left[w t\left(\bar{x}^{n}\right)\right]=\left(n p_{1}, \cdots, n p_{d}\right)$. The weak law of large numbers states that for any $\epsilon>0$,

$$
\left.\lim _{n \rightarrow \infty} p\left[\| \frac{1}{n} w t\left(\bar{x}^{n}\right)-E\left[w t\left(\bar{x}^{n}\right)\right)\right] \|_{1}>\epsilon\right]=0
$$

where for $f: \mathcal{Z} \rightarrow \mathbb{R}^{d}$, define $\|f\|_{1}=\sum_{z \in \mathcal{Z}}|f(z)|$. In our case, $\mathcal{Z}=\mathcal{X}^{n}$. 
Now simply throw out all strings $\bar{x}^{n}$ with $\left\|\frac{1}{n}\left(w t\left(\bar{x}^{n}\right)-E\left[w t\left(\bar{x}^{n}\right)\right)\right]\right\|_{1}>\epsilon$, and take $\mathcal{Y}(n)$ of size

$$
\begin{aligned}
|\mathcal{Y}(n)| & =\#\left\{\bar{x}^{n} \mid\left\|\frac{1}{n}\left(w t\left(\bar{x}^{n}\right)-E\left[w t\left(\bar{x}^{n}\right)\right)\right]\right\|_{1}<\epsilon\right\} \\
& =\sum_{\substack{\bar{x}^{n} \mid \\
\left\|\frac{1}{n}\left(w t\left(\bar{x}^{n}\right)-E\left[w t\left(\bar{x}^{n}\right)\right)\right]\right\|_{1}<\epsilon}}\left(\begin{array}{c}
n \\
w t\left(\bar{x}^{n}\right)
\end{array}\right) .
\end{aligned}
$$

If $\epsilon$ is small, the multinomial coefficients appearing will all be very close to

$$
\left(\begin{array}{c}
n \\
n p_{1}, \cdots, n p_{d}
\end{array}\right)
$$

and for what follows, one can take the crude approximation

$$
|\mathcal{Y}(n)| \leq \operatorname{poly}(n)\left(\begin{array}{c}
n \\
n p_{1}, \cdots, n p_{d}
\end{array}\right)
$$

(recall that $d$ is fixed).

Taking logarithms, the right hand side of $(10)$ becomes $n H(\bar{p})+O(\log (n))$. Thus

$$
\frac{1}{n} \log |\mathcal{Y}(n)| \leq H(\bar{p})+o(1)
$$

and $\operatorname{Rate}(\bar{p}) \leq H(\bar{p})$.

Theorem 5.1. [Sha48] Rate $(\bar{p})=H(\bar{p})$.

The full proof uses the strong law of large numbers.

5.2. Transmission over noisy channels. Say symbols $x$ are transmitted over a channel subject to noise, and symbols $y$ are received so one may or may not have $y=x$. Intuitively, if the noise is small, with some redundancy it should be possible to communicate accurate messages most of the time. Let Rate denote the maximal possible rate of transmission. In a noiseless channel this is just $H\left(p_{\mathcal{X}}\right)$, but now we must subtract off something to account for the uncertainty that, upon receiving $y$, that it was the signal sent. This something will be the conditional entropy: Recall the conditional probability of $i$ occurring given knowledge that $j$ occurs (assuming $p(j)>0): p_{\mathcal{X} \mid \mathcal{Y}}(i \mid j)=\frac{p_{\mathcal{X}, \mathcal{Y}}(i, j)}{p_{\mathcal{Y}}(j)}\left(\right.$ also recall $\left.p_{\mathcal{Y}}(j)=\sum_{i} p_{\mathcal{X}, \mathcal{Y}}(i, j)\right)$. Define the conditional entropy

$$
H\left(\bar{p}_{\mathcal{Y}} \mid \bar{p}_{\mathcal{X}}\right):=-\sum_{i, j} p_{\mathcal{X}, \mathcal{Y}}(i, j) \log p_{\mathcal{Y} \mid \mathcal{X}}(j \mid i)
$$

Note that

$$
H\left(\bar{p}_{\mathcal{Y}} \mid \bar{p}_{\mathcal{X}}\right)=H\left(\bar{p}_{\mathcal{X}, \mathcal{Y}}\right)-H\left(\bar{p}_{\mathcal{X}}\right)
$$

or equivalently $H\left(\bar{p}_{\mathcal{X}, \mathcal{Y}}\right)=H\left(\bar{p}_{\mathcal{X}}\right)+H\left(\bar{p}_{\mathcal{Y}} \mid \bar{p}_{\mathcal{X}}\right)$, the uncertainty of $p_{\mathcal{X}, \mathcal{Y}}$ is the uncertainty of $p_{\mathcal{X}}$ plus the uncertainty of $p_{\mathcal{Y}}$ given $p_{\mathcal{X}}$.

In particular $H\left(\bar{p}_{\mathcal{Y}}\right) \geq H\left(\bar{p}_{\mathcal{Y}} \mid \bar{p}_{\mathcal{X}}\right)$, i.e., with extra knowledge, our uncertainty about $p_{\mathcal{Y}}$ cannot increase, and decreases unless $p_{\mathcal{X}}$ and $p_{\mathcal{Y}}$ are independent). 
5.2.1. Capacity of a noisy channel. Define the capacity of a noisy channel to be the maximum rate over all possible probability distributions on the source:

$$
\text { Cap }:=\max _{q_{\mathcal{X}}}\left(H\left(q_{\mathcal{X}}\right)-H\left(q_{\mathcal{X}} \mid p_{\mathcal{Y}}\right)\right) \text {. }
$$

Shannon [Sha48] proves that Cap lives up to its name: if the entropy of a discrete channel is below Cap then there exists an encoding $\bar{p}$ of the source such that information can be transmitted over the channel with an arbitrarily small frequency of errors. The basic idea is the same as the noiseless case, however there is a novel feature that now occurs frequently in complexity theory arguments - that instead of producing an algorithm to find the efficient encoding, Shannon showed that a random choice of encoding will work.

After presenting the proof, Shannon remarks: "An attempt to obtain a good approximation to ideal coding by following the method of the proof is generally impractical. ... Probably this is no accident but is related to the difficulty of giving an explicit construction for a good approximation to a random sequence". To my knowledge, this is the first time that the difficulty of "finding hay in a haystack" (phrase due to Howard Karloff) is mentioned in print. This problem is central to complexity: for example, Valiant's algebraic version of $\mathbf{P} \neq \mathbf{N P}$ can be phrased as the problem of finding a sequence of explicit polynomials that are difficult to compute, while it is known that a random sequence is indeed difficult to compute. According to A. Wigderson, the difficulty of writing down random objects problem was also explicitly discussed by Erdös, in the context of random graphs, at least as early as 1947, in relation to his seminar paper [Erd47]. This paper, along with [Sha48] gave rise to the now ubiquitous probabilistic method in complexity theory.

\section{ReFormulation OF QUANTUM MeChaniCS}

I discuss two inconveniences about our formulation of the postulates of quantum mechanics, leading to a formulation of the postulates in terms of density operators.

6.1. Partial measurements. A measurement of a state $|\psi\rangle=\sum z_{I}|I\rangle$ was defined a procedure that gives us $I=\left(i_{1}, \cdots, i_{n}\right) \in\{0,1\}^{n}$ with probability $\left|z_{I}\right|^{2}$. But in our algorithms, this is not what happened: we were working not in $\left(\mathbb{C}^{2}\right)^{\otimes n}$, but $\left(\mathbb{C}^{2}\right)^{\otimes n+m}$ where there were $m$ "workspace" qubits we were not interested in measuring. So our measurement was more like the projections onto the spaces $|I\rangle \otimes\left(\mathbb{C}^{2}\right)^{\otimes m}$. I now define this generalized notion of measurement.

To make the transition, first observe that $\left|z_{I}\right|^{2}=\left\langle\psi\left|\Pi_{I}\right| \psi\right\rangle$, where $\Pi_{I}:\left(\mathbb{C}^{2}\right)^{\otimes n} \rightarrow \mathbb{C}|I\rangle$ is the orthogonal projection onto the line spanned by $|I\rangle$.

Now say we are only interested in the first $n$ bits of a system of $n+m$ bits, and want to know the probability a measurement gives rise to some $I$ represented by a vector $|I\rangle \in\left(\mathbb{C}^{2}\right)^{\otimes n}$, but we have $|\psi\rangle \in\left(\mathbb{C}^{2}\right)^{\otimes n+m}$. Adopt the notation $|\phi\rangle\langle\psi|:=| \phi\rangle \otimes\langle\psi|$. Then the probability of obtaining $|I\rangle$ given $|\psi\rangle$ is

$$
\begin{aligned}
p(|I\rangle|| \psi\rangle) & =\sum_{J \in\{0,1\}^{m}} p(|\psi\rangle,|I J\rangle) \\
& =\sum_{J}\langle\psi \mid I J\rangle\langle I J \mid \psi\rangle \\
& =\langle\psi|\left(|I\rangle\langle I| \otimes \operatorname{Id}_{\left.\left(\mathbb{C}^{2}\right)^{\otimes m}\right)|\psi\rangle}\right. \\
& =\left\langle\psi\left|\Pi_{\mathcal{M}}\right| \psi\right\rangle
\end{aligned}
$$


where $\Pi_{\mathcal{M}}:\left(\mathbb{C}^{2}\right)^{\otimes n+m} \rightarrow|I\rangle \otimes\left(\mathbb{C}^{2}\right)^{\otimes m}=: \mathcal{M}$ is the orthogonal projection operator. Then $p(|I\rangle \mid$ $|\psi\rangle)=\left\langle\psi\left|\Pi_{\mathcal{M}}\right| \psi\right\rangle$. With this definition, one can allow $\mathcal{M} \subset \mathcal{H}$ to be any linear subspace, which will simplify our measurements. (Earlier, if we wanted to measure the probability of a non-basis state, we had to change bases before measuring.)

One may think of projection operators as representing outside interference of a quantum system, like adding a filter to beams being sent that destroy states not in $\mathcal{M}$. Recall that in classical probability, one has the identity:

$$
p\left(M_{1} \cup M_{2}\right)=p\left(M_{1}\right)+p\left(M_{2}\right)-p\left(M_{1} \cap M_{2}\right) .
$$

The quantum analog is false in general: Let $\mathcal{H}=\mathbb{C}^{2}, \mathcal{M}_{1}=\mathbb{C}|0\rangle$ and $\mathcal{M}_{2}=\mathbb{C}(|0\rangle+|1\rangle)$ Let $|\psi\rangle=\alpha|0\rangle+\beta|1\rangle$ with $|\alpha|^{2}+|\beta|^{2}=1$. Then (and in general) $p\left(\operatorname{span}\left\{\mathcal{M}_{1}, \mathcal{M}_{2}\right\}\right) \neq p\left(\mathcal{M}_{1}\right)+$ $p\left(\mathcal{M}_{2}\right)-p\left(\mathcal{M}_{1} \cap \mathcal{M}_{2}\right)$.

However, one can recover (12) if the projection operators commute:

Proposition 6.1. If $\Pi_{\mathcal{M}_{1}} \Pi_{\mathcal{M}_{2}}=\Pi_{\mathcal{M}_{2}} \Pi_{\mathcal{M}_{1}}$ then $p\left(\operatorname{span}\left\{\mathcal{M}_{1}, \mathcal{M}_{2}\right\}\right)=p\left(\mathcal{M}_{1}\right)+p\left(\mathcal{M}_{2}\right)-p\left(\mathcal{M}_{1} \cap\right.$ $\left.\mathcal{M}_{2}\right)$.

6.2. Mixing classical and quantum probability. A typical situation in probability is as follows: you want a cookie, but can't make up your mind which kind, so you decide to take one at random from the cookie jar to eat. However when you open the cupboard, you find there are two different cookie jars $H$ and $T$, each with a different distribution of cookies, say $P_{H}$ and $P_{T}$. You decide to flip a coin to decide which jar and say your coin is biased with probability $p$ for heads (choice $H$ ). The resulting probability distribution is

$$
p P_{H}+(1-p) P_{T} .
$$

Let's encode this scenario with vectors. Classically, if vectors corresponding to $P_{H}, P_{T}$ are respectively $v_{H}, v_{T}$, the new vector is $p v_{H}+(1-p) v_{T}$. The probability of drawing a chocolate chip $(\mathrm{CC})$ cookie is $p P_{H}(C C)+(1-p) P_{T}(C C)=p v_{H, C C}+(1-p) v_{T, C C}$.

But what should one take in generalized probability (where one uses the $\ell_{2}$ norm instead of the $\ell_{1}$ norm)? Given $\left|\psi_{A}\right\rangle=\sum z_{I}|I\rangle,\left|\psi_{B}\right\rangle=\sum w_{J}|J\rangle$, we want to make a measurement that gives us $p\left|z_{C C}\right|^{2}+(1-p)\left|w_{C C}\right|^{2}$. Unfortunately $\left|p z_{C C}+(1-p) w_{C C}\right|^{2} \neq p\left|z_{C C}\right|^{2}+(1-p)\left|w_{C C}\right|^{2}$ in general. To fix this problem I will enlarge the notion of state and further modify our notion of measurement.

Our problem comes from having a mixture of $\ell_{1}$ and $\ell_{2}$ norms. The fix will be to rewrite $|\psi\rangle$ in a way that the $\ell_{2}$ norm becomes an $\ell_{1}$ norm. That is, I construct an object that naturally contains the squares of the norms of the coefficients of $\left|\psi_{A}\right\rangle$. Consider the endomorphism $\left|\psi_{A}\right\rangle\left\langle\psi_{A}\right|=$ $\sum_{I, J} z_{I} \bar{z}_{J}|I\rangle\langle J|$. It is rank one, and in our standard basis its diagonal entries are the quantities we want.

To measure them, let $\Pi_{J}$ denote the projection onto the $J$-th coordinate. Then

$$
\operatorname{trace}\left(\Pi_{J}\left|\psi_{A}\right\rangle\left\langle\psi_{A}\right|\right)=\left|z_{A, J}\right|^{2}
$$

is the desired quantity.

Now back to our cookie jars, set

$$
\rho=p\left|\psi_{A}\right\rangle\left\langle\psi_{A}|+(1-p)| \psi_{B}\right\rangle\left\langle\psi_{B}\right|
$$

and observe that

$$
\operatorname{trace}\left(\Pi_{J} \rho\right)=p\left|z_{A, J}\right|^{2}+(1-p)\left|z_{B, J}\right|^{2}
$$


as desired.

Given a finite set of states $\left\{\left|\psi_{1}\right\rangle, \cdots,\left|\psi_{s}\right\rangle\right\}$, with $p\left(\left|\psi_{i}\right\rangle\right)=p_{i}$, and $\sum_{i} p_{i}=1$, set $\rho=\sum_{k} p_{k}\left|\psi_{k}\right\rangle\left\langle\psi_{k}\right| \epsilon$ $\operatorname{End}(\mathcal{H})$. Note that $\rho$ has the properties

(1) $\rho=\rho^{\dagger}$, i.e., $\rho$ is Hermitian,

(2) $\forall|\eta\rangle,\langle\eta|\rho| \eta\rangle \geq 0$, i.e., $\rho$ is positive,

(3) $\operatorname{trace}(\rho)=1$.

This motivates the following definition:

Definition 6.2. An operator $\rho \in \operatorname{End}(\mathcal{H})$ satisfying $1,2,3$ above is called a density operator.

Note that a density operator that is diagonal in the standard basis of $\mathbb{C}^{d}$ corresponds to a probability distribution on $\{1, \cdots, d\}$, so the definition includes classical probability as well as our old notion of state (which are the rank one density operators). The set of density operators is invariant under the induced action of $\mathbf{U}(\mathcal{H})$ on $\operatorname{End}(\mathcal{H})$.

Different scenarios can lead to the same density operator. However, two states with the same density operator are physically indistinguishable.

\subsection{Reformulation of the postulates of quantum mechanics.}

Postulate 1. Associated to any isolated physical system is a Hilbert space $\mathcal{H}$, call the state space. The system is described by its density operator $\rho \in \operatorname{End}(\mathcal{H})$.

Postulate 2. The evolution of an isolated system is described by the action of unitary operators on $\rho$.

Postulate 3. (POVM) Measurements correspond to a collection of projection operators $\Pi_{\mathcal{M}_{j}}$ such that $\sum_{k} \Pi_{\mathcal{M}_{k}}=\operatorname{Id}_{\mathcal{H}}$. The probability that $\rho$ is in measured in state $\mathcal{M}_{j}$ is trace $\left(\Pi_{\mathcal{M}_{j}} \rho\right)$.

Sometimes it is convenient to allow more general measurements:

Postulate 3'. Projective measurements correspond to a collection of Hermitian operators $X_{j} \in$ End $\mathcal{H}$ such that $\sum_{k} X_{k}=\operatorname{Id}_{\mathcal{H}}$. The probability that $\rho$ is in measured in state $X_{j}$ is $\operatorname{trace}\left(X_{j} \rho\right)$.

Postulate 4 regarding composite systems is unchanged.

Remark 6.3. Note that for $A \in \operatorname{End} \mathcal{H}=\mathcal{H}^{*} \otimes \mathcal{H}$, trace $(A)$ is the image of $A$ under the contraction map $\mathcal{H}^{*} \otimes \mathcal{H} \rightarrow \mathbb{C},\langle v|\otimes| w\rangle \mapsto\langle v \mid w\rangle$. For $A \in \operatorname{End}\left(\mathcal{H}_{1} \otimes \mathcal{H}_{2}\right)=\left(\mathcal{H}_{1}^{*} \otimes \mathcal{H}_{2}^{*}\right) \otimes\left(\mathcal{H}_{1} \otimes \mathcal{H}_{2}\right)$, define the partial trace trace $\mathcal{H}_{1}(A)$ to be the image of $A$ under the contraction $\mathcal{H}_{1}^{*} \otimes \mathcal{H}_{2}^{*} \otimes \mathcal{H}_{1} \otimes \mathcal{H}_{2} \rightarrow \mathcal{H}_{2}^{*} \otimes \mathcal{H}_{2}$ given by $\langle\phi|\otimes\langle\psi|\otimes| v\rangle \otimes| w\rangle \mapsto\langle\phi \mid v\rangle\langle\psi|\otimes| w\rangle=\langle\phi \mid v\rangle|w\rangle\langle\psi|$.

6.4. Expectation and the uncertainty principle. Let $A \in \operatorname{End}(H)$ be a Hermitian operator with eigenvalues $\lambda_{1}, \cdots, \lambda_{k}$ and eigenspaces $\mathcal{M}_{j}$. If our system is in state $\rho$, one can consider $A$ as a random variable that takes the value $\lambda_{j}$ with probability trace $\left(\Pi_{\mathcal{M}_{j}} \rho\right)$.

The expectation of a random variable $X: \mathcal{X} \rightarrow \mathbb{R}$ is $E[X]:=\sum_{j \in \mathcal{X}} X(j) p(j)$.

If a system is in state $\rho$, the expectation of a Hermitian operator $A \in \operatorname{End}(H)$ is $\operatorname{trace}(A \rho)$ because $E[A]=\sum_{\lambda_{j}} \lambda_{j} \operatorname{trace}\left(\Pi_{\mathcal{M}_{j}} \rho\right)=\operatorname{trace}\left(\left(\sum_{\lambda_{j}} \lambda_{j} \Pi_{\mathcal{M}_{j}}\right) \rho\right)=\operatorname{trace}(A \rho)$. 
One way mathematicians describe the famous Heisenberg uncertainty principle is that it is impossible to localize both a function and its Fourier transform. Another interpretation comes from probability:

First note that given a random variable, or Hermitian operator $X$, one can replace it with an operator of mean zero $\hat{X}:=X-E(X \rho)$ Id. For notational convenience, I state the uncertainty principle for such shifted operators.

The variance $\operatorname{var}(X)$ of a random variable is $\operatorname{var}(X)=E[X-E(X)]^{2}$. The standard deviation $\sigma(X)=\sqrt{\operatorname{var}(X)}$ of $X$ is a measure of the failure of the corresponding probability distribution to be concentrated at a point, i.e., failure of the induced probability distribution to have a certain outcome.

Proposition 6.4. Let $X, Y$ be Hermitian operators of mean zero, corresponding to observables on a system in state $\rho$, let Then

$$
\sigma(X) \sigma(Y) \geq \frac{|\operatorname{trace}([X, Y] \rho)|}{2} .
$$

The uncertainty principle says that the failure of two Hermitian operators to commute lower bounds the product of their uncertainties. In particular, if they do not commute, neither can give rise to a classical (certain) measurement. It is a consequence of the Cauchy-Schwarz inequality.

\subsection{Pure and mixed states.}

Definition 6.5. Let $\rho \in \operatorname{End}(\mathcal{H})$ be a density operator. If $\operatorname{rank}(\rho)=1$, i.e. $\rho=|\xi\rangle\langle\xi|, \rho$ is called a pure state, and otherwise it is called a mixed state.

The partial trace of a pure state can be a mixed state. For example, if $\rho=|\psi\rangle\langle\psi|$ with $\psi=$ $\frac{1}{\sqrt{2}}(|00\rangle+|11\rangle) \in \mathcal{H}_{1} \otimes \mathcal{H}_{2}$, then $\operatorname{trace}_{\mathcal{H}_{2}}(\rho)=\frac{1}{2}(|0\rangle\langle 0|+| 1\rangle\langle 1|)$.

The following proposition shows that one could avoid density operators altogether by working on a larger space:

Proposition 6.6. An arbitrary mixed state $\rho \in \operatorname{End}(\mathcal{H})$ can be represented as the partial trace trace $\mathcal{H}^{\prime}|\psi\rangle\langle\psi|$ of a pure state in $\operatorname{End}\left(\mathcal{H} \otimes \mathcal{H}^{\prime}\right)$ for some Hilbert space $\mathcal{H}^{\prime}$. In fact, one can always take $\mathcal{H}^{\prime}=\mathcal{H}^{*}$.

Given a density operator $\rho \in \operatorname{End}(\mathcal{H})$, there is a well defined density operator $\sqrt{\rho} \in \operatorname{End}(\mathcal{H})$ whose eigenvectors are the same as for $\rho$, and whose eigenvalues are the positive square roots of the eigenvalues of $\rho$. To prove the proposition, given $\rho \in \mathcal{H} \otimes \mathcal{H}^{*}$, consider $|\sqrt{\rho}\rangle\langle\sqrt{\rho}| \in \operatorname{End}\left(\mathcal{H} \otimes \mathcal{H}^{*}\right)$. Then $\rho=\operatorname{trace}_{\mathcal{H}^{*}}(|\sqrt{\rho}\rangle\langle\sqrt{\rho}|)$. A pure state whose partial trace is $\rho$ is called a purification of $\rho$.

\section{Communication across a Quantum Channel}

Now instead of having a source $\mathcal{X}^{\times n}$ our "source" is $\mathcal{H}^{\otimes n}$, where one can think of $\mathcal{H}^{\otimes n}=\mathcal{H}_{A}^{\otimes n}$, and Alice will "transmit" a state to Bob, and instead of a probability distribution $p$ one has a density operator $\rho$.

What is a quantum channel? It should be a linear map sending $\rho \in \operatorname{End}\left(\mathcal{H}_{A}\right)$ to some $\Phi(\rho) \epsilon$ $\operatorname{End}\left(\mathcal{H}_{B}\right)$. 
First consider the special case $\mathcal{H}_{A}=\mathcal{H}_{B}$. One should allow coupling with an auxiliary system, i.e.,

$$
\rho \mapsto \rho \otimes \sigma \in \operatorname{End}\left(\mathcal{H}_{A} \otimes \mathcal{H}_{C}\right) .
$$

One should also allow the state $\rho \otimes \sigma$ to evolve in $\operatorname{End}\left(\mathcal{H}_{A} \otimes \mathcal{H}_{C}\right)$, i.e., be acted upon by an arbitrary $U \in \mathbf{U}\left(\mathcal{H}_{A} \otimes \mathcal{H}_{C}\right)$. Finally one should allow measurements, i.e., tracing out the $\mathcal{H}_{C}$ part. In summary, a quantum channel $\mathcal{H}_{A} \rightarrow \mathcal{H}_{A}$ is a map of the form $\rho \mapsto \operatorname{trace} \mathcal{H}_{C}\left(U(\rho \otimes \sigma) U^{-1}\right)$. More generally to go from $\mathcal{H}_{A}$ to $\mathcal{H}_{B}$, one needs to allow isometries as well. Such maps are the completely positive trace preserving maps (CPTP), where a map $\Lambda$ is completely positive if $\Lambda \otimes \operatorname{Id}_{\mathcal{H}_{E}}$ is positive for all $\mathcal{H}_{E}$.

We seek an encoder $\mathcal{E}$ and decoder $\mathcal{D}$ and a compression space $\mathcal{H}_{0 n}$ :

$$
\mathcal{H}^{\otimes n} \stackrel{\mathcal{E}}{\rightarrow} \mathcal{H}_{0 n}=\left(\mathbb{C}^{2}\right)^{\otimes n R} \stackrel{\mathcal{D}}{\rightarrow} \mathcal{H}^{\otimes n}
$$

with $R$ as small as possible such that $\mathcal{E} \circ \mathcal{D}\left(\rho^{\otimes n}\right)$ converges to $\rho^{\otimes n}$ as $n \rightarrow \infty$. To determine $R$, we need a quantum version of entropy.

Definition 7.1. The von Neumann entropy of a density operator $\rho$ is $H(\rho)=-\operatorname{trace}(\rho \log (\rho))$.

Here $\log (\rho)$ is defined as follows: write $\rho$ in terms of its eigenvectors and eigenvalues, $\rho=$ $\sum_{j} \lambda_{j}\left|\psi_{j}\right\rangle\left\langle\psi_{j}\right|$, then $\log (\rho)=\sum_{j} \log \left(\lambda_{j}\right)\left|\psi_{j}\right\rangle\left\langle\psi_{j}\right|$.

If $\rho=\sum_{j} \lambda_{j}\left|\psi_{j}\right\rangle\left\langle\psi_{j}\right|$, then $H(\rho)=-\sum_{j} \lambda_{j} \log \left(\lambda_{j}\right)$ so if $\rho$ is classical (i.e., diagonal), one obtains the Shannon entropy.

Proposition 7.2. The von Neumann entropy has the following properties:

(1) $H(\rho) \geq 0$ with equality if and only if $\rho$ is pure.

(2) Let $\operatorname{dim} \mathcal{H}=d$. Then $H(\rho) \leq \log (d)$ with equality if and only if $\rho=\frac{1}{d} \operatorname{Id}_{\mathcal{H}}$.

(3) If $\rho=|\psi\rangle\langle\psi| \in \operatorname{End}\left(\mathcal{H}_{A} \otimes \mathcal{H}_{B}\right)$, then $H\left(\rho_{A}\right)=H\left(\rho_{B}\right)$, where $\rho_{A}=\operatorname{trace}_{\mathcal{H}_{B}}(\rho) \in \operatorname{End}\left(\mathcal{H}_{A}\right)$.

Theorem 7.3. [Sch95]/The quantum noiseless channel theorem] Let $(\mathcal{H}, \rho)$ be an i.i.d. quantum source. If $R>H(\rho)$, then there exists a reliable compression scheme of rate $R$. That is, there exists a compression space $\mathcal{H}_{0 n}$, of dimension $2^{n R}$, and encoder $\mathcal{E}: \mathcal{H}^{\otimes n} \rightarrow \mathcal{H}_{0 n}$ and a decoder $\mathcal{D}: \mathcal{H}_{0 n} \rightarrow \mathcal{H}^{\otimes n}$ such that $\mathcal{D} \circ \mathcal{E}\left(\rho^{\otimes n}\right)$ converges to $\rho^{\otimes n}$ as $n \rightarrow \infty$. If $R<H(\rho)$, then any compression scheme is unreliable.

\section{More on von Neumann entropy and its variants}

First for the classical case, define the relative entropy $H(\bar{p}|| \bar{q}):=-\sum p_{i} \log \frac{q_{i}}{p_{i}}=-H(\bar{p})-\sum_{i} p_{i} \log \left(q_{i}\right)$. It is zero when $\bar{p}=\bar{q}$ and is otherwise positive. Define the relative von Neumann entropy $H(\rho \| \sigma):=\operatorname{trace}(\rho \log (\rho))-\operatorname{trace}(\rho \log (\sigma))$. It shares the positivity property of its classical cousin: [Kle] $H(\rho \| \sigma) \geq 0$ with equality if and only if $\rho=\sigma$.

von Neumann Entropy is non-decreasing under projective measurements: Let $\Pi_{i}$ be a complete set of orthogonal projectors, set $\rho^{\prime}=\sum_{i} \Pi_{i} \rho \Pi_{i}$. Then $H\left(\rho^{\prime}\right) \geq H(\rho)$ with equality if and only if $\rho^{\prime}=\rho$.

Here and in what follows $\rho_{A B}$ is a density operator on $\mathcal{H}_{A} \otimes \mathcal{H}_{B}$ and $\rho_{A}=\operatorname{trace} \mathcal{H}_{B}\left(\rho_{A B}\right), \rho_{B}=$ $\operatorname{trace}_{\mathcal{H}_{A}}\left(\rho_{A B}\right)$ are respectively the induced density operators on $\mathcal{H}_{A}, \mathcal{H}_{B}$. 
von Neumann entropy is sub-additive: $H\left(\rho_{A B}\right) \leq H\left(\rho_{A}\right)+H\left(\rho_{B}\right)$ with equality if and only if $\rho_{A B}=\rho_{A} \otimes \rho_{B}$. It also satisfies a triangle inequality: $H\left(\rho_{A B}\right) \geq\left|H\left(\rho_{A}\right)-H\left(\rho_{B}\right)\right|$.

Recall the conditional Shannon entropy is defined to be $H\left(\bar{p}_{\mathcal{X}} \mid \bar{p}_{\mathcal{Y}}\right)=-\sum_{i, j} p_{\mathcal{X} \times \mathcal{Y}}(i, j) \log p_{\mathcal{X} \mid \mathcal{Y}}(i \mid j)$, the entropy of $p_{\mathcal{X}}$ conditioned on $y=j$, averaged over $\mathcal{Y}$. It is not clear how to "condition" one density matrix on another, so one needs a different definition. Recall that Shannon entropy satisfies $H\left(\bar{p}_{\mathcal{X}} \mid \bar{p}_{\mathcal{Y}}\right)=H\left(\bar{p}_{\mathcal{X} \times \mathcal{Y}}\right)-H\left(\bar{p}_{\mathcal{Y}}\right)$, and the right hand side of this expression does make sense for density operators, so define, for $\rho_{A B}$ a density operator on $\mathcal{H}_{A} \otimes \mathcal{H}_{B}$,

$$
H\left(\rho_{A} \mid \rho_{B}\right):=H\left(\rho_{A B}\right)-H\left(\rho_{B}\right) .
$$

Note that $H\left(\rho_{A} \mid \rho_{B}\right)$ is a function of $\rho_{A B}$, as $\rho_{B}=\operatorname{trace}_{\mathcal{H}_{A}} \rho_{A B}$.

WARNING: it is possible that the conditional von Neumann entropy is negative as it is possible that $H\left(\rho_{B}\right)>H\left(\rho_{A B}\right)$. Consider the following example: Let $|\psi\rangle=\frac{1}{\sqrt{2}}(|00\rangle+|11\rangle) \in \mathcal{H}_{A} \otimes \mathcal{H}_{B}$. Then $\rho_{A}=\frac{1}{2} \operatorname{Id}_{\mathcal{H}_{A}}=\frac{1}{2}(|0\rangle\langle 0|+| 1\rangle\langle 1|)$ so $H\left(\rho_{A}\right)=1$, but $H(|\psi\rangle\langle\psi|)=0$ because $|\psi\rangle\langle\psi|$ is pure.

However, vestiges of positivity are true in the quantum case:

Theorem 8.1 (Strong sub-additivity). Let $\rho_{A B C}$ be a density operator on $\mathcal{H}_{A} \otimes \mathcal{H}_{B} \otimes \mathcal{H}_{C}$. Then

$$
H\left(\rho_{C} \mid \rho_{A}\right)+H\left(\rho_{C} \mid \rho_{B}\right) \geq 0
$$

and

$$
H\left(\rho_{A B C}\right)-\left[H\left(\rho_{A B}\right)+H\left(\rho_{B C}\right)\right]+H\left(\rho_{B}\right) \geq 0 .
$$

\section{Entanglement and LOCC}

We have seen several ways that entanglement is a resource already for the space $\mathcal{H}_{A} \otimes \mathcal{H}_{B}=$ $\mathbb{C}^{2} \otimes \mathbb{C}^{2}$ : given a shared $|e p r\rangle=\frac{1}{\sqrt{2}}(|00\rangle+|11\rangle)$, one can transport two bits of classical information using only one qubit ("super dense coding") and one can also transmit one qubit of quantum information from Alice to Bob by sending two classical bits ("teleportation").

9.1. LOCC. Assume several different laboratories can communicate classically, have prepared some shared states in advance, and can perform unitary and projection operations on their parts of the states, as was the situation for quantum teleportation. More precisely, make the following assumptions:

- $\mathcal{H}=\mathcal{H}_{1} \otimes \cdots \otimes \mathcal{H}_{n}$, and the $\mathcal{H}_{j}$ share an entangled state $|\psi\rangle$. Often one will just have $\mathcal{H}=\mathcal{H}_{A} \otimes \mathcal{H}_{B}$ and $|\psi\rangle=\alpha|00\rangle+\beta|11\rangle$.

- The laboratories can communicate classically.

- Each laboratory is allowed to perform unitary and measurement operations on their own spaces.

The above assumptions are called $L O C C$ for "local operations and classical communication". It generalizes the set-up for teleportation $\S 3.2$.

Restrict to the case $\mathcal{H}=\mathcal{H}_{A} \otimes \mathcal{H}_{B}$, each of dimension two. I will use $\mid$ epr $\rangle$ as a benchmark for measuring the quality of entanglement.

We will not be concerned with a single state $|\psi\rangle$, but the tensor product of many copies of it, $|\psi\rangle^{\otimes n} \in\left(\mathcal{H}_{A} \otimes \mathcal{H}_{B}\right)^{\otimes n}$. "How much" entanglement does $|\psi\rangle^{\otimes n}$ have? An answer is given in $\S 9.4$. 
To gain insight as to which states can be produced via LOCC from a given density operator, return to the classical case. For the classical cousin of LOCC, by considering diagonal density operators, we see we should allow alteration of a probability distribution by permuting the $p_{j}$ (permutation matrices are unitary), and more generally averaging our probability measure under some probability measure on elements of $\mathfrak{S}_{d}$ (the classical cousin of a projective measurement), i.e., we should allow

$$
\bar{p} \mapsto \sum_{\sigma \in \mathfrak{S}_{d}} q_{\sigma} \mu(\sigma) \bar{p}
$$

where $\mu: \mathfrak{S}_{d} \rightarrow G L_{d}$ is the representation, and $q$ is a probability distribution on $\mathfrak{S}_{d}$.

This is because the unitary and projection local operators allowed amount to

$$
\rho \mapsto \sum_{j=1}^{k} p_{j} U_{j} \rho U_{j}^{-1}
$$

where the $U_{j}$ are unitary and $p$ is a probability distribution on $\{1, \cdots, k\}$ for some finite $k$.

9.2. A partial order on probability distributions compatible with entropy. Shannon entropy is non-increasing under an action of the form (17). The partial order on probability distributions determined by (17) is the dominance order:

Definition 9.1. Let $x, y \in \mathbb{R}^{d}$, write $x^{\downarrow}$ for $x$ re-ordered such that $x_{1} \geq x_{2} \geq \cdots \geq x_{d}$. Write $x<y$ if for all $k \leq d, \sum_{j=1}^{k} x_{j}^{\downarrow} \leq \sum_{j=1}^{k} y_{j}^{\downarrow}$.

Note that if $p$ is a probability distribution concentrated at a point, then $\bar{q}<\bar{p}$ for all probability distributions $q$, and if $p$ is such that $p_{j}=\frac{1}{d}$ for all $j$, then $\bar{p}<\bar{q}$ for all $q$, and more generally the dominance order is compatible with the entropy in the sense that $\bar{p}<\bar{q}$ implies $H(\bar{p}) \geq H(\bar{q})$.

Recall that a matrix $D \in M a t_{d \times d}$ is doubly stochastic if $D_{i j} \geq 0$ and all column and row sums equal one. Let $\mathcal{D} \mathcal{S}_{d} \subset M a t_{d \times d}$ denote the set of doubly stochastic matrices. G. Birkoff [Bir46] showed $\mathcal{D} \mathcal{S}_{d}=\operatorname{conv}\left(\mu\left(\mathfrak{S}_{d}\right)\right)$, and Hardy-Littlewood-Polya [HLP52] showed $\{x \mid x<y\}=\mathcal{D} \mathcal{S}_{d} \cdot y$.

9.3. A reduction theorem. The study of LOCC is potentially unwieldy because there can be numerous rounds of local operations and classical communication, making it hard to model. The following result eliminates this problem:

Proposition 9.2. If $|\psi\rangle \in \mathcal{H}_{A} \otimes \mathcal{H}_{B}$ can be transformed into $|\phi\rangle$ by LOCC, then it can be transformed to $|\phi\rangle$ by the following sequence of operations:

(1) Alice performs a single measurement with operators $\Pi_{M_{j}}$.

(2) She sends the result of her measurement (some j) to Bob classically.

(3) Bob performs a unitary operation on his system.

The key point is that for any vector spaces $V, W$, an element $f \in V \otimes W$, may be considered as a linear map $W^{*} \rightarrow V$. In our case, $\mathcal{H}_{B}^{*} \simeq \mathcal{H}_{B}$ so $|\psi\rangle$ induces a linear map $\mathcal{H}_{B} \rightarrow \mathcal{H}_{A}$ which gives us the mechanism to transfer Bob's measurements to Alice.

Now I can state the main theorem on LOCC:

Theorem 9.3. [Nie99] $|\psi\rangle \leadsto|\phi\rangle$ by LOCC if and only if $\operatorname{singvals}(|\psi\rangle)<\operatorname{singvals}(|\phi\rangle)$. 
9.4. Entanglement distillation (concentration) and dilution. To compare the entanglement resources of two states $|\phi\rangle$ and $|\psi\rangle$, consider $|\phi\rangle^{\otimes m}$ for large $m$ with the goal of determining the largest $n=n(m)$ such that $|\phi\rangle^{\otimes m}$ may be degenerated to $|\psi\rangle^{\otimes n}$ via LOCC. Due to the approximate and probabilistic nature of quantum computing, relax this to degenerating $|\phi\rangle^{\otimes m}$ to a state that is close to $|\psi\rangle^{\otimes n}$.

There is a subtlety for this question worth pointing out. Teleportation was defined in such a way that Alice did not need to know the state she was teleporting, but for distillation and dilution, she will need to know that its right singular vectors are standard basis vectors. More precisely, if she is in possession of $|\psi\rangle=\sqrt{p_{1}}\left|v_{1}\right\rangle \otimes|1\rangle+\sqrt{p_{2}}\left|v_{2}\right\rangle \otimes|2\rangle$, she can teleport the second half of it to Bob if they share $|e p r\rangle \in \mathcal{H}_{A} \otimes \mathcal{H}_{B}$. More generally, if she is in possession of $|\psi\rangle=\sum_{j=1}^{d} \sqrt{p_{j}}\left|v_{j}\right\rangle \otimes|j\rangle \in \mathcal{H}_{A^{\prime}} \otimes \mathcal{H}_{A^{\prime \prime}}$, she can teleport it to Bob if they share enough EPR states. In most textbooks, Alice is assumed to possess states whose singular vectors are $|j j\rangle$ 's and I will follow that convention here. Similarly, if $|\psi\rangle=\sum_{j=1}^{d} \sqrt{p_{j}}|j j\rangle \in \mathcal{H}_{A} \otimes \mathcal{H}_{B}$, I discuss how many shared EPR states they can construct from a shared $|\psi\rangle^{\otimes m}$.

Define the entanglement cost $E_{C}(\psi)$ to be $\inf _{m} \frac{n(m)}{m}$ where $n(m)$ copies of $\psi$ can be constructed from $|e p r\rangle^{\otimes m}$ by LOCC with error going to zero as $m \rightarrow \infty$. Similarly, define the entanglement value, or distillable entanglement $E_{V}(\psi)$ to be $\sup _{m} \frac{n(m)}{m}$ where $n(m)$ copies of $\mid$ epr $\rangle$ can be constructed with diminishing error from $|\psi\rangle^{\otimes m}$ by LOCC. One has $E_{V}(\psi)=E_{C}(\psi)=H(|\psi\rangle\langle\psi|)$.

Remark 9.4. In classical computation one can reproduce information, but this cannot be done with quantum information in general. This is because the map $|\psi\rangle \mapsto|\psi\rangle \otimes|\psi\rangle$, called the Veronese map in algebraic geometry, is not a linear map. This observation is called the no cloning theorem in the quantum literature. However, one can define a linear map, e.g., $\mathbb{C}^{2} \rightarrow \mathbb{C}^{2} \otimes \mathbb{C}^{2}$ that duplicates basis vectors, i.e., $|0\rangle \mapsto|0\rangle \otimes|0\rangle$ and $|1\rangle \mapsto|1\rangle \otimes|1\rangle$. But then of course $\alpha|0\rangle+\beta|1\rangle \mapsto$ $\alpha|0\rangle \otimes|0\rangle+\beta|1\rangle \otimes|1\rangle \neq(a|0\rangle+\beta|1\rangle)^{\otimes 2}$.

For mixed states $\rho$ on $\mathcal{H}_{A} \otimes \mathcal{H}_{B}$, one can still define $E_{C}(\rho)$ and $E_{V}(\rho)$, but there exist examples where they differ, so there is not a canonical measure of entanglement. A wish list of what one might want from an entanglement measure $E$ :

- Non-increasing under LOCC.

- If $\rho$ is a product state, i.e., $\rho=\left|\phi_{A}\right\rangle\left\langle\phi_{A}|\otimes| \psi_{B}\right\rangle\left\langle\psi_{B}\right|$, then $E(\rho)=0$.

The two conditions together imply any state constructible from a product state by LOCC should also have zero entanglement. Hence the following definition:

Definition 9.5. A density operator $\rho \in \operatorname{End}\left(\mathcal{H}_{1} \otimes \cdots \otimes \mathcal{H}_{n}\right)$ is separable if $\rho=\sum_{i} p_{i} \rho_{i, 1} \otimes \cdots \otimes \rho_{i, n}$, where $\rho_{i, \alpha} \in \operatorname{End}\left(\mathcal{H}_{\alpha}\right)$ are density operators, $p_{i} \geq 0$, and $\sum_{i} p_{i}=n$. If $\rho$ is not separable, $\rho$ is entangled.

Definition 9.6. An entanglement monotone $E$ is a function on density operators on $\mathcal{H}_{A} \otimes \mathcal{H}_{B}$ that is non-increasing under LOCC.

An example of an entanglement monotone different from $E_{V}, E_{C}$ useful for general density operators is defined in [CW04]. 


\section{SLOCC}

An entanglement measure appealing to geometers is SLOCC (stochastic local operations and classical communication) defined originally in [BPR $\left.{ }^{+} 00\right]$, which asks if $|\psi\rangle \in \mathcal{H}_{1} \otimes \cdots \otimes \mathcal{H}_{d}$ is in the same $S L\left(\mathcal{H}_{1}\right) \times \cdots \times S L\left(\mathcal{H}_{d}\right)$ orbit as $|\phi\rangle \in \mathcal{H}_{1} \otimes \cdots \otimes \mathcal{H}_{d}$. If one relaxes this to orbit closure, then it amounts to being able to convert $|\psi\rangle$ to $|\phi\rangle$ with positive probability. While appealing, and while there is literature on SLOCC, given the probabilistic nature of quantum computing, its use appears to be limited to very special cases, where the orbit structure is understood (e.g., $\left.d \leq 4, \operatorname{dim} \mathcal{H}_{j}=2\right)$.

\section{TENSOR NETWORK STATES}

Physically, entanglement is more likely when the particles are closer together, so if we have an arrangement of electrons, say on a circle, as in Figure 2:

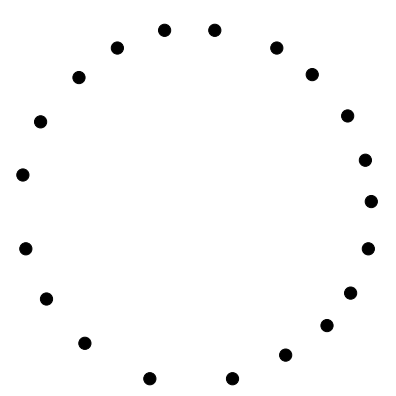

FiguRE 2. Electrons arranged on a circle

It is highly improbable that the electrons will share entanglement with any but their nearest neighbors. This is fortuitous, because if one is dealing with thousands of electrons and would like to describe their joint state, a priori one would have to work with a vector space of dimension $2^{n}$, with $n$ in the thousands, which is not feasible. The practical solution to this problem is to define a subset of $\left(\mathbb{C}^{2}\right)^{\otimes n}$ of reasonable dimension (e.g. $\left.O(n)\right)$ consisting of the probable states.

For example, say the isolated system consists of electrons arranged along a line as in 11.

and we only want to allow electrons to be entangled with their nearest neighbors. This leads to the notion of Matrix Product States (MPS): draw a graph reflecting this geometry, with a vertex for each electron. To each vertex, attach edges going from the electron's vertex to those of its nearest neighbors, and add an additional edge not attached to anything else (these will be called physical edges). If our space is $\mathcal{H}_{1} \otimes \cdots \otimes \mathcal{H}_{n}$, then, assuming vertex $j$ has two neighbors, attach two auxiliary vector spaces, $E_{j-1}, E_{j}^{*}$, and a tensor $T_{j} \in \mathcal{H}_{j} \otimes E_{j-1} \otimes E_{j}^{*}$. If we are on a line, to vertex one, we just attach $T_{1} \in \mathcal{H}_{1} \otimes E_{1}^{*}$, and similarly, to vertex $n$ we attach $T_{n} \in \mathcal{H}_{n} \otimes E_{n-1}$. Now consider the tensor product of all the tensors

$$
T_{1} \otimes \cdots \otimes T_{n} \in\left(\mathcal{H}_{1} \otimes E_{1}^{*}\right) \otimes\left(\mathcal{H}_{2} \otimes E_{1} \otimes E_{2}\right) \otimes \cdots \otimes\left(\mathcal{H}_{n-1} \otimes E_{n-2} \otimes E_{n-1}^{*}\right) \otimes\left(\mathcal{H}_{n} \otimes E_{n-1}\right)
$$

Assume each $E_{j}$ has dimension $k$. We can contract these to obtain a tensor $T \in \mathcal{H}_{1} \otimes \cdots \otimes \mathcal{H}_{n}$. If $k=1$, we just obtain the product states. As we increase $k$, we obtain a steadily larger subset of $\mathcal{H}_{1} \otimes \cdots \otimes \mathcal{H}_{n}$. The claim is that the tensors obtainable in this fashion (for some $k$ determined by the physical setup) are exactly those locally entangled states that we seek. 


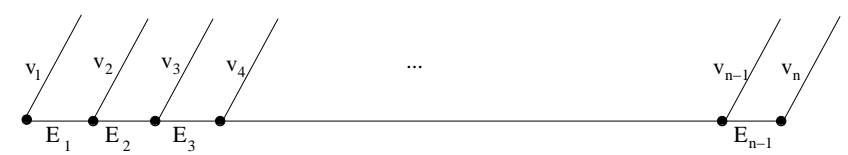

For the circle, the only difference in the construction is to make the result periodic, so $T_{1} \in$ $\mathcal{H}_{1} \otimes E_{n} \otimes E_{1}^{*}$ and $T_{n} \in \mathcal{H}_{n} \otimes E_{n-1} \otimes E_{n}^{*}$.

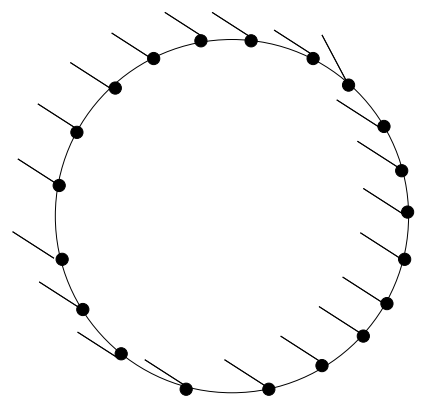

Sometimes for applications (e.g. translation invariant systems on the circle) one requires the same tensor be placed at each vertex. If the tensor is $\sum_{i, j, \alpha} T_{i, j, \alpha}\langle i|\otimes| j\rangle \otimes v_{\alpha}$, the resulting tensor is $\sum T_{i_{1}, i_{2}, \alpha_{1}} T_{i_{2}, i_{3}, \alpha_{2}} \cdots T_{i_{n}, i_{1}, \alpha_{n}} v_{\alpha_{1}} \otimes \cdots \otimes v_{\alpha_{n}}$.

For a second example, consider electrons arranged in a rectangular array (or on a grid on a torus), where each vertex is allowed to interact with its four nearest neighbors.

Assume we place the same tensor at each vertex. If our grid is $n \times n$ and periodic, we obtain a $\operatorname{map}\left(\mathbb{C}^{k}\right)^{\otimes 4} \otimes \mathbb{C}^{d} \rightarrow\left(\mathbb{C}^{d}\right)^{\otimes n^{2}}$.

Definition 11.1. Let $\Gamma$ be a directed graph with vertices $v_{\alpha}$ and two kinds of edges: "physical" edges $e_{i}$, that are attached to a single vertex, and "auxiliary" (or entanglement) edges $e_{s}$ between two vertices. Associate to each physical edge a vector space $V_{i}$ (or perhaps better $\mathcal{H}_{i}$ ), and to each auxiliary edge a vector space $E_{s}$, of dimension $\mathbf{e}_{s}$. Let $\overline{\mathbf{e}}=\left(\mathbf{e}_{1}, \cdots, \mathbf{e}_{f}\right)$ denote the vector of these dimensions. A tensor network state associated to $\left(\Gamma,\left\{V_{i}\right\}, \overline{\mathbf{e}}\right)$ is a tensor $T \in V_{1} \otimes \cdots \otimes V_{n}$ obtained as follows: To each vertex $v_{\alpha}$, associate a tensor

$$
T_{\alpha} \in \otimes_{i \in \alpha} V_{i} \otimes_{s \in i n(\alpha)} E_{s}^{*} \otimes_{t \in \text { out }(\alpha)} E_{t} .
$$

Here $i n(\alpha)$ are the edges going into vertex $\alpha$ and $\operatorname{out}(\alpha)$ are the edges going out of the vertex. The tensor network state associated to this configuration is $T:=\operatorname{contr}\left(T_{1} \otimes \cdots \otimes T_{g}\right) \in V_{1} \otimes \cdots \otimes V_{n}$. Let $T N S\left(\Gamma, V_{1} \otimes \cdots \otimes V_{n}, \mathbf{e}\right) \subset V_{1} \otimes \cdots \otimes V_{n}$ denote the set of tensor network states.

Example 11.2. Let $\Gamma$ be:

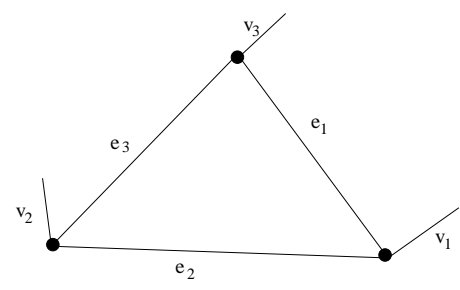

Then

$$
\begin{aligned}
T N S\left(\Gamma, V_{1} \otimes V_{2} \otimes V_{3}, \overline{\mathbf{e}}\right) & =T N S\left(\Gamma,\left(E_{1}^{*} \otimes E_{2}\right) \otimes\left(E_{2}^{*} \otimes E_{3}\right) \otimes\left(E_{3}^{*} \otimes E_{1}\right), \overline{\mathbf{e}}\right) \\
& =\operatorname{End}\left(V_{1}\right) \times \operatorname{End}\left(V_{2}\right) \times \operatorname{End}\left(V_{3}\right) \cdot M_{\left\langle\mathbf{e}_{1}, \mathbf{e}_{2}, \mathbf{e}_{3}\right\rangle} .
\end{aligned}
$$


Here $M_{\left\langle\mathbf{e}_{1}, \mathbf{e}_{2}, \mathbf{e}_{3}\right\rangle}$ is the matrix multiplication tensor, for $A \in \operatorname{Mat}_{\mathbf{e}_{1} \times \mathbf{e}_{2}}, B \in$ Mat $_{\mathbf{e}_{2} \times \mathbf{e}_{3}}, C \in$ Mat $_{\mathbf{e} 3 \times \mathbf{e} 1},(A, B, C) \mapsto \operatorname{trace}(A B C)$. Let $e_{1}, \cdots, e_{\mathbf{e}_{1}}$ be a basis of $E_{1}, f_{1}, \cdots, f_{\mathbf{e}_{2}}$ be a basis of $E_{2}$, and $g_{1}, \cdots, e_{\mathbf{e}_{3}}$ be a basis of $E_{3}$.

There are many open questions about tensor network states: what are the algebraic varieties one obtains from the Zariski closure of a given TNS?

\section{REPRESENTATION THEORY IN QUANTUM INFORMATION THEORY}

Natural projection operators on $V^{\otimes d}$ are determined by representation theory. I review the relevant representation theory and then apply it to describe the solution to the quantum marginal problem.

12.1. Review of relevant representation theory. (Isomorphism classes of) irreducible representations of the permutation group $\mathfrak{S}_{d}$ are indexed by partitions of $d$, write $[\pi]$ for the $\mathfrak{S}_{d^{-}}$ module corresponding to the partition $\pi$. The irreducible polynomial representations of $G L(V)$ are indexed by partitions $\pi=\left(p_{1}, \cdots, p_{\ell(\pi)}\right)$ with $\ell(\pi) \leq \operatorname{dim} V$. Write $S_{\pi} V$ for the corresponding $G L(V)$-module.

Theorem 12.1 (Schur-Weyl duality). As a $G L(V) \times \mathfrak{S}_{d}$-module,

$$
V^{\otimes d}=\bigoplus_{|\pi|=d} S_{\pi} V \otimes[\pi]
$$

Let $P_{\pi}: V^{\otimes d} \rightarrow S_{\pi} V \otimes[\pi]$ denote the $G L(V) \times \mathfrak{S}_{d}$-module projection operator.

One is often interested in decompositions of a module under the action of a subgroup. For example $S^{d}(V \otimes W)$ is an irreducible $G L(V \otimes W)$-module, but as a $G L(V) \times G L(W)$-module it has the decomposition, called the Cauchy formula,

$$
S^{d}(V \otimes W)=\oplus_{|\pi|=d} S_{\pi} V \otimes S_{\pi} W .
$$

We will be particularly interested in the decomposition of $S^{d}(U \otimes V \otimes W)$ as a $G L(U) \times G L(V) \times$ $G L(W)$-module. An explicit formula for this decomposition is not known. Write

$$
S^{d}(U \otimes V \otimes W)=\bigoplus_{|\pi|,|\mu|,|\nu|=d}\left(S_{\pi} U \otimes S_{\mu} V \otimes S_{\nu} W\right)^{\oplus k_{\pi, \mu, \nu}} .
$$

The numbers $k_{\pi, \nu, \mu}$ that record the multiplicities are called Kronecker coefficients. They have several additional descriptions. For example, $S_{\pi}(V \otimes W)=\bigoplus_{|\mu|,|\nu|=d}\left(S_{\mu} V \otimes S_{\nu} W\right)^{\oplus k_{\pi, \mu, \nu}}$, and $k_{\pi, \mu, \nu}=\operatorname{dim}([\pi] \otimes[\mu] \otimes[\mu])^{\mathfrak{S}_{d}}=\operatorname{mult}([d],[\pi] \otimes[\mu] \otimes[\nu])=\operatorname{mult}([\pi],[\mu] \otimes[\nu])$.

12.2. Quantum marginals and projections onto isotypic subspaces of $\mathcal{H}^{\otimes d}$. In this section I address the question: what are compatibility conditions on density operators $\rho$ on $\mathcal{H}_{A} \otimes \mathcal{H}_{B}, \rho^{\prime}$ on $\mathcal{H}_{A}$ and $\rho^{\prime \prime}$ on $\mathcal{H}_{B}$ such that $\rho^{\prime}=\operatorname{trace}_{\mathcal{H}_{B}}(\rho), \rho^{\prime \prime}=\operatorname{trace} \mathcal{H}_{A}(\rho)$ ? As you might expect by now, compatibility will depend only on the spectra of the operators.

Above I discussed representations of the general linear group $G L(V)$ where $V$ is a complex vector space. In quantum theory, one is interested in representations on the unitary group $\mathbf{U}(\mathcal{H})$ on a Hilbert space $\mathcal{H}$. The unitary group is a real Lie group, not a complex Lie group, because complex conjugation is not a complex linear map. It is a special case of a general fact about representations of a maximal compact subgroups of complex Lie groups have the same 
representation theory as the the original group, so in particular the decomposition of $\mathcal{H}^{\otimes d}$ as a $\mathbf{U}(\mathcal{H})$-module coincides with its decomposition as a $G L(\mathcal{H})$-module.

For a partition $\pi=\left(p_{1}, \cdots, p_{d}\right)$ of $d$, introduce the notation $\bar{\pi}=\left(\frac{p_{1}}{d}, \cdots, \frac{p_{d}}{d}\right)$ which is a probability distribution on $\{1, \cdots, d\}$.

Theorem 12.2. [CM06] Let $\rho_{A B}$ be a density operator on $\mathcal{H}_{A} \otimes \mathcal{H}_{B}$. Then there exists a sequence $\left(\pi_{j}, \mu_{j}, \nu_{j}\right)$ of triples of partitions such that $k_{\pi_{j}, \mu_{j}, \nu_{j}} \neq 0$ for all $j$ and

$$
\begin{aligned}
& \lim _{j \rightarrow \infty} \bar{\pi}_{j}=\operatorname{spec}\left(\rho_{A B}\right) \\
& \lim _{j \rightarrow \infty} \bar{\mu}_{j}=\operatorname{spec}\left(\rho_{A}\right) \\
& \lim _{j \rightarrow \infty} \bar{\nu}_{j}=\operatorname{spec}\left(\rho_{B}\right) .
\end{aligned}
$$

Theorem 12.3. [Kly04] Let $\rho_{A B}$ be a density operator on $\mathcal{H}_{A} \otimes \mathcal{H}_{B}$ such that $\operatorname{spec}\left(\rho_{A B}\right)$, $\operatorname{spec}\left(\rho_{A}\right)$ and $\operatorname{spec}\left(\rho_{B}\right)$ are all rational vectors. Then there exists an integer $M>0$ such that

$$
k_{M \operatorname{spec}\left(\rho_{A}\right), M \operatorname{spec}\left(\rho_{B}\right), M \operatorname{spec}\left(\rho_{C}\right)} \neq 0 .
$$

Theorem 12.4. [Kly04] Let $\pi, \mu, \nu$ be partitions of $d$ with $k_{\pi, \mu, \nu} \neq 0$ and satisfying $\ell(\pi) \leq m n$, $\ell(\mu) \leq m$, and $\ell(\nu) \leq n$. Then there exists a density operator $\rho_{A B}$ on $\mathbb{C}^{n} \otimes \mathbb{C}^{m}=\mathcal{H}_{A} \otimes \mathcal{H}_{B}$ with $\operatorname{spec}\left(\rho_{A B}\right)=\bar{\pi}, \operatorname{spec}\left(\rho_{A}\right)=\bar{\mu}$, and $\operatorname{spec}\left(\rho_{B}\right)=\bar{\nu}$.

Klyatchko's proofs are via co-adjoint orbits and vector bundles on flag varieties, while the proof of Christandl-Mitchison is information-theoretic in flavor.

Recall the relative entropy $H(\bar{p} \| \bar{q})=-\sum_{i} p_{i} \log \frac{q_{i}}{p_{i}}$, which may be thought of as measuring how close $p, q$ are because it is non-negative, and zero if and only if $p=q$. A key step in the Christandl-Mitchison proof is the following theorem:

Theorem 12.5. [KW01] Let $\rho \in \operatorname{End}(\mathcal{H})$ be a density operator, where $\operatorname{dim} \mathcal{H}=n$. Let $|\pi|=d$ and let $P_{\pi}: \mathcal{H}^{\otimes d} \rightarrow S_{\pi} \mathcal{H} \otimes[\pi]$ be the projection operator. Then

$$
\operatorname{trace}\left(P_{\pi} \rho^{\otimes d}\right) \leq(d+1)^{\left(\begin{array}{c}
n \\
2
\end{array}\right)} e^{-d H(\bar{\pi} \| \operatorname{spec}(\rho))} .
$$

A key step of the proof is that the projection of $e_{I}$ to $S_{\pi} V \otimes[\pi]$ is nonzero if and only if $w t\left(e_{I}\right)<\pi$.

Note that one can use quantum theory to deduce representation-theoretic consequences: $k_{\mu, \nu, \pi} \neq$ 0 implies $H(\bar{\pi}) \leq H(\bar{\mu})+H(\bar{\nu}), H(\bar{\mu}) \leq H(\bar{\pi})+H(\bar{\nu})$, and $H(\bar{\nu}) \leq H(\bar{\mu})+H(\bar{\pi})$.

Let $\operatorname{Spec}_{m, n, m n}$ denote the set of admissible triples $\left(\operatorname{spec}\left(\rho_{A}\right), \operatorname{spec}\left(\rho_{B}\right), \operatorname{spec}\left(\rho_{A B}\right)\right.$ and $K R O N_{m, n, m n}$ the triples $(\bar{\mu}, \bar{\nu}, \bar{\pi})$ of normalized partitions $(\mu, \nu, \pi)$ with $\ell(\mu) \leq m, \ell(\nu) \leq n, \ell(\pi) \leq m n$ and $k_{\pi, \mu, \nu} \neq 0$.

The theorems above imply:

$$
\operatorname{Spec}_{m, n, m n}=\overline{K R O N_{m, n, m n}} .
$$

In particular, $S p e c_{m, n, m n}$ is a convex polytope.

Acknowledgements. I thank the organizers of the International workshop on Quantum Physics and Geometry, especially Alessandra Bernardi, who also co-organized an intensive summer class on Quantum computing and quantum information theory that I gave June-July 2017. I also thank L. Chiantini, F. Gesmundo, F. Holweck, and G. Ottaviani for useful comments on a draft of this article. 


\section{REFERENCES}

[Aar13] Scott Aaronson, Quantum computing since Democritus, Cambridge University Press, Cambridge, 2013. MR 3058839

[AB09] Sanjeev Arora and Boaz Barak, Computational complexity, Cambridge University Press, Cambridge, 2009, A modern approach. MR 2500087 (2010i:68001)

[BCHW16] F. G. S. L. Brandao, M. Christandl, A. W. Harrow, and M. Walter, The Mathematics of Entanglement, ArXiv e-prints (2016).

[Bel64] J.S. Bell, On the einstein-podolsky-rosen paradox, Physics 1 (1964), 195-200.

[Bir46] Garrett Birkhoff, Three observations on linear algebra, Univ. Nac. Tucumán. Revista A. 5 (1946), 147-151. MR 0020547

$\left[\mathrm{BPR}^{+} 00\right]$ Charles H. Bennett, Sandu Popescu, Daniel Rohrlich, John A. Smolin, and Ashish V. Thapliyal, Exact and asymptotic measures of multipartite pure-state entanglement, Phys. Rev. A 63 (2000), 012307.

[CHSH69] John F. Clauser, Michael A. Horne, Abner Shimony, and Richard A. Holt, Proposed experiment to test local hidden-variable theories, Phys. Rev. Lett. 23 (1969), 880-884.

[CM06] Matthias Christandl and Graeme Mitchison, The spectra of quantum states and the Kronecker coefficients of the symmetric group, Comm. Math. Phys. 261 (2006), no. 3, 789-797. MR 2197548

[CW04] Matthias Christandl and Andreas Winter, "Squashed entanglement": an additive entanglement measure, J. Math. Phys. 45 (2004), no. 3, 829-840. MR 2036165

[EPR35] A. Einstein, B. Podolsky, and N. Rosen, Can quantum-mechanical description of physical reality be considered complete?, Phys. Rev. 47 (1935), 777-780.

[Erd47] P. Erdös, Some remarks on the theory of graphs, Bull. Amer. Math. Soc. 53 (1947), $292-294$. MR 0019911

[HLP52] G. H. Hardy, J. E. Littlewood, and G. Pólya, Inequalities, Cambridge, at the University Press, 1952, 2d ed. MR 0046395

[Kle] O. Klein.

[Kly04] A. Klyachko, Quantum marginal problem and representations of the symmetric group, preprint arXiv:quant-ph/0409113v1 (2004).

[KSV02] A. Yu. Kitaev, A. H. Shen, and M. N. Vyalyi, Classical and quantum computation, Graduate Studies in Mathematics, vol. 47, American Mathematical Society, Providence, RI, 2002, Translated from the 1999 Russian original by Lester J. Senechal. MR 1907291

[KW01] M. Keyl and R. F. Werner, Estimating the spectrum of a density operator, Phys. Rev. A (3) 64 (2001), no. 5, 052311, 5. MR 1878924

[Lan17] J.M. Landsberg, Quantum computation and information: Notes for fall 2017 tamu class, available at http://www.math.tamu.edu/ jml/quantumnotes.pdf (2017).

[NC00] Michael A. Nielsen and Isaac L. Chuang, Quantum computation and quantum information, Cambridge University Press, Cambridge, 2000. MR MR1796805 (2003j:81038)

[Nie99] M. A. Nielsen, Conditions for a class of entanglement transformations, P H Y S I C A L R E V I E W L E T T E R S 83 (1999), 436-439.

[Sch95] Benjamin Schumacher, Quantum coding, Phys. Rev. A (3) 51 (1995), no. 4, 2738-2747. MR 1328824

[Sha48] C. E. Shannon, A mathematical theory of communication, Bell System Tech. J. 27 (1948), 379-423, 623-656. MR 0026286 